\title{
Service-Outage-Based Power and Rate Control for Poisson Fading Channels
}

\author{
Kaushik Chakraborty, Member, IEEE, Subhrakanti Dey, Senior Member, IEEE, and \\ Massimo Franceschetti, Member, IEEE
}

\begin{abstract}
A single-input-single-output (SISO) Poisson fading channel with perfect channel state information (CSI) at the transmitter and the receiver is considered. For a fixed basic rate $r_{0}$, a service outage occurs when the instantaneous transmission rate falls below the rate $r_{0}$. The objective of this paper is to maximize the expected transmission rate subject to peak and average transmitter power constraints and a constraint on the service outage probability. The optimal power allocation scheme is shown to be a combination of the ergodic capacity-achieving power allocation and the outage capacity-achieving power allocation schemes with a randomization between the two deterministic schemes in a boundary set. This randomization is not necessary when the channel fade distribution is continuous. By combining the concepts of ergodic and outage capacity, the proposed optimal scheme judiciously resolves the conflicting objectives of high expected transmission rate and low outage probability.
\end{abstract}

Index Terms-Adaptive transmission, channel state information (CSI), ergodic capacity, outage capacity, Poisson fading channel, service outage.

\section{INTRODUCTION}

$\mathbf{F}$ REE-SPACE OPTICS (FSO) has gained much popularity in the recent years as a low-cost, high-data rate communication strategy. It has been proposed as a viable solution to the last mile problem, i.e., the final leg of delivering connectivity from a communication provider to a customer, especially in the urban setting [19]. The many benefits of wireless optical systems include rapid deployment time, inexpensive components, seamless wireless extension of the optical fiber backbone, immunity from radio-frequency (RF) interference, high security, and lack of licensing regulations. Consequently, FSO

Manuscript received February 06, 2008; revised September 05, 2008. Current version published April 22, 2009. This work was supported in part by the California Institute of Telecommunication and Information Technology and by the National Science Foundation under Grants CCF-0635048 and CAREER CNS-0546235. The material in this paper was presented in part at the IEEE International Symposium on Information Theory, Toronto, ON, Canada, July 2008.

K. Chakraborty is with Qualcomm Inc., San Diego, CA 92121 USA (e-mail: kauchaks@qualcomm.com).

S. Dey is with the ARC Special Research Center for Ultra-Broadband Information Networks (CUBIN), Department of Electrical and Electronic Engineering, University of Melbourne, Parkville, Vic. 3010, Australia (e-mail: sdey@unimelb.edu.au).

M. Franceschetti is with the Department of Electrical and Computer Engineering, University of California, San Diego, La Jolla, CA 92093 USA (e-mail: massimo@ece.ucsd.edu).

Communicated by G. Taricco, Associate Editor for Communications.

Color versions of Figures 4 and 7 in this paper are available online at http:// ieeexplore.ieee.org.

Digital Object Identifier 10.1109/TIT.2009.2015995 communication has received much recent attention in the literature [2]-[4], [7], [8], [11], [12], [20], [21]. In free-space optical communication links, atmospheric turbulence causes random fluctuations in the refractive index of air at optical wavelengths, which in turn cause random fluctuations in the intensity of a propagating optical signal [9]. Hence, the FSO communication channel can be modeled as a slowly varying fading channel with occasional deep fades that can affect millions of consecutive bits [2], [7].

In time-varying channels, e.g., wireless RF communication, dynamic allocation of resources, e.g., transmit power and information rate, can lead to improved communication performance over fixed resource allocation schemes. The communication performance, which can be characterized in terms of the rate of information transferred from the transmitter to the receiver, is often application specific. For example, for delay-sensitive applications the capacity versus outage probability is an important design parameter whereas for nonreal-time applications the expected information rate or ergodic capacity is an appropriate measure of communication performance. Therefore, different classes of applications will benefit from adaptive transmission schemes that provide better communication performance with respect to the specific design parameters. For a comprehensive review in the context of RF communication, see [16]. One popular technique to combat the detrimental effects of fading is to estimate the time-varying channel conditions at the transmitter and the receiver and use the channel state information (CSI) to dynamically adapt transmit power and rate according to the existing channel conditions. In this paper, we assume that perfect CSI is available to the transmitter and the receiver, so that the transmitter can dynamically adjust its power level and instantaneous rate based on perfect CSI. While ergodic capacity-achieving power allocation leads to maximum average throughput [6], for real-time applications in slow fading environments, it is not a good performance measure since averaging over a large number of channel states can lead to unacceptable delay. On the other hand, outage capacity-achieving power allocation for a given outage probability guarantees a constant basic rate for channel states not in outage [1]. There are several variable-rate real-time applications for which different levels of quality of service, quantified in terms of the allocated rate for each of these levels, can be guaranteed for different applications. For example, in an application with simultaneous voice and data transmissions, as soon as a basic rate $r_{0}$ has been guaranteed for the voice service, any excess available rate can be used to transmit data in a best effort fashion such that the long-term average rate is maximized for the delay-insensitive 
data applications. Motivated by these practical considerations, in [14] and [15], Luo et al. studied variable rate adaptive transmission schemes subject to a maximum outage probability constraint on a basic rate requirement in a slow fading RF environment. The notions of ergodic and outage capacity were judiciously combined in a service-outage-based problem formulation, where the long-term average rate was maximized subject to a basic rate requirement in addition to the customary transmitter average power constraint. In this paper, we apply similar ideas in the context of a free-space optical fading channel.

In this paper, we consider a shot-noise limited singleinput-single-output (SISO) free-space intensity modulation direct detection (IM/DD) optical channel in which information is transmitted by modulating the intensity of the transmitted signal, and individual photon arrivals are observed at the direct detection receiver. In [2], a block fading channel model [10] was proposed to account for the slowly varying nature of optical fade in FSO communication channels. The channel fade is assumed to remain unvarying for a coherence interval of fixed duration $T_{\mathrm{c}}$ time units, and change across successive such intervals in an independent and identically distributed (i.i.d.) manner. In this paper, we assume the same fading channel model. The IM/DD optical block fading channel with Poisson receiver statistics is succinctly called the Poisson fading channel. In [2], the ergodic capacity of the SISO Poisson fading channel was derived, and properties of the capacity-achieving power allocation schemes were characterized. The multiple-input-multiple-output (MIMO) Poisson fading channel was studied in [3], [4], and [7]. While upper and lower bounds on ergodic and outage capacity were derived in [7], exact expressions for ergodic and outage capacity were obtained in [3] and [4], respectively. A complete characterization of optimal power allocation schemes that achieve outage capacity was also derived in [4]. However, a complete characterization of optimal power allocation schemes that achieve ergodic capacity for the MIMO Poisson fading channel still remains open [3]; a few special cases have been solved. In this paper, we restrict ourselves to the SISO case, and assume that perfect CSI is available to the transmitter and the receiver.

It is important to note that our model ignores bandwidth limitations associated with practical transmitter and receiver devices. We also incorporate the effects of signal shot noise and background radiation into a constant rate dark current parameter. These simplifying assumptions lead to an idealized channel model that is amenable to an exact analysis. Other channel models have been proposed in the literature, which describe the background radiation as additive white Gaussian (cf., e.g., [8], [11], [12], and [21]), or consider spectrally constrained transmitted signals (cf., e.g., [17] and [18]).

The remainder of this paper is organized as follows. In Section II, a formal description of the service-outage-based power and rate allocation problem for Poisson fading channel is outlined. The optimal power allocation scheme is derived in Section III, and several interesting properties are identified in Section IV. A numerical example with lognormal fading channel model is provided in Section V. Finally, Section VI contains a discussion of the main results and directions for future research.

\section{PROBLEM Formulation}

The following notation will be used consistently throughout the paper. Random variables are denoted by uppercase letters and their realizations are denoted by lowercase letters. The following letters will be exclusively used to denote specific system parameters: $A$ denotes peak power, $\lambda_{0}$ denotes the background noise rate, $\sigma$ denotes the average-to-peak power ratio, $\epsilon$ denotes the probability of outage, and $r_{0}$ denotes the basic rate. The instantaneous rate (mutual information) is denoted by the function $I$ and the maximum rate (capacity) is denoted by $C$. A deterministic power allocation scheme is denoted by $\gamma$, and a probabilistic power allocation scheme by $\Gamma$. A superscript $(\cdot)^{*}$ usually denotes an optimal solution, and a subscript denotes a special definition, e.g., $\gamma_{\text {erg }}^{*}$ is the optimal ergodic capacity-achieving (deterministic) power allocation. Sets are denoted by calligraphic font, e.g., $\mathcal{S}_{0}\left(r_{0}\right)$ denotes the outage floor set corresponding to a basic rate of $r_{0}$. The complement of a set is denoted by $(\cdot)^{c}$ and the closure by $(\bar{\cdot})$.

We consider a service-outage-based power and rate allocation problem for the SISO Poisson fading channel when the transmitter and the receiver are provided with perfect CSI. A general class of probabilistic power allocation schemes are considered, akin to the approach proposed in [15] for the parallel Gaussian fading channel.

A block schematic diagram of the channel model is given in Fig. 1. For a given $\mathbb{R}^{+}$-valued transmitted signal $x^{\infty}=\{x(t), t \geq 0\}$, the corresponding received signal at the channel output $Y^{\infty}=\{Y(t), t \geq 0\}$ is a Poisson counting process $(\mathrm{PCP})$ with rate

$$
\Lambda(t)=S\left[\left[\frac{t}{T_{\mathrm{c}}}\right]\right] x(t)+\lambda_{0}, \quad t \geq 0
$$

where $\left\{S[k], k \in \mathbb{Z}^{+}\right\}$is the i.i.d. $\mathbb{R}^{+}$-valued random fade in the $k$ th coherence interval $\left((k-1) T_{\mathrm{c}}, k T_{\mathrm{c}}\right]$, and $\lambda_{0} \geq 0$ is the background noise (dark current) rate which is assumed to be constant. In (1), we denote by $\lceil x\rceil$ the smallest integer greater than or equal to $x$. With $[0, T]$ being the interval of transmission and reception, the input $x^{T}=\{x(t), 0 \leq t \leq T\}$ is proportional to the transmitted optical power, and satisfies peak and average power constraints

$$
\begin{gathered}
0 \leq x(t) \leq A, \quad 0 \leq t \leq T \\
\frac{1}{T} \int_{0}^{T} x(t) d t \leq \sigma A
\end{gathered}
$$

where the peak power $A>0$ and the ratio of average-to-peak power $\sigma, 0 \leq \sigma \leq 1$, are fixed.

In [2], a single-letter characterization of ergodic capacity of the SISO Poisson fading channel was derived and the properties of the capacity-achieving power allocation scheme were identified. It was shown that a binary ON-OFF keying (OOK) signaling scheme with arbitrarily fast intertransition times ${ }^{1}$ can achieve capacity. The probability that the transmit aperture, i.e., the transmitting photoemitter, will remain $\mathrm{ON}$ as a function of

\footnotetext{
${ }^{1}$ In OOK signaling, a state transition occurs when the transmit aperture shifts from the ON state to the OFF state, or vice versa. The minimum time interval between two consecutive state transitions is the intertransition time.
} 


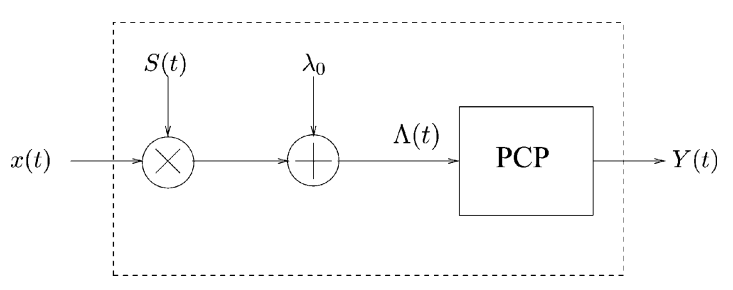

Fig. 1. Poisson fading channel.

the instantaneous channel fade is denoted by $\gamma: \mathbb{R}^{+} \rightarrow[0,1]$, and can be seen as the conditional duty cycle of the transmitted signal, conditioned on the channel fade. The instantaneous mutual information of the SISO Poisson fading channel, as a function of the channel fade $s$ and the duty cycle $\gamma$ is given by [2]

$$
I(\gamma, s)=\gamma \zeta\left(s A, \lambda_{0}\right)-\zeta\left(\gamma s A, \lambda_{0}\right)
$$

where we define ${ }^{2}$

$$
\zeta(x, y) \triangleq(x+y) \log (x+y)-y \log y, \quad x, y \geq 0 .
$$

A single-letter characterization of the ergodic capacity can be posed in terms of an optimization problem as in [2, Th. 1]. The optimal power allocation scheme, which was outlined in $[2, \mathrm{Th}$. 2], can be interpreted as the allocation of the conditional transmitter duty cycle that maximizes the expected information rate subject to the peak and average power constraints. The outage capacity of MIMO Poisson fading channels was characterized in [4], again in terms of an optimization problem involving the conditional duty cycles of the transmit apertures. In this paper, we consider a service-outage-based power allocation problem, which combines the concepts of ergodic and outage capacity, and aims to resolve the conflicting objectives of achieving high expected transmission rate and low outage probability.

When OOK signaling scheme is used, the transmitted power is directly proportional (with a scaling factor equal to the peak transmit power $A$ ) to the probability that the transmit aperture is ON, or equivalently to the duty cycle of the transmit aperture. Therefore, the duty cycle $\gamma$ can be viewed as a power allocation parameter for the transmit aperture, and henceforth, we use the terms duty cycle and power allocation interchangeably. A probabilistic power allocation is a random variable (rv) $\Gamma$ with a conditional probability density function (pdf) $f_{\Gamma \mid S}$. Each realization $\gamma$ of $\Gamma$ corresponds to a particular deterministic power allocation (which corresponds to the instantaneous duty cycle of the transmit aperture) given a particular channel realization $s$, and $f_{\Gamma \mid S}(\gamma \mid s)$ specifies the proportion of time the power allocation $\gamma$ is used when the channel state is $s$. In a probabilistic power allocation scheme, each realization of the power allocation is associated with a coding scheme; a service outage occurs when the code rate is less than the basic rate $r_{0}$ specified by the application [15]. For each channel fade, a service outage occurs with a nonzero probability, since among the multiple codes associated with the probabilistic power allocation scheme, some code rates

\footnotetext{
${ }^{2}$ All logarithms are natural logarithms.
}

can exceed or fall below $r_{0}$. For notational convenience, we denote by $\Gamma(\cdot)$ a probabilistic power allocation scheme with conditional pdf $f_{\Gamma \mid S}$, and a deterministic power allocation scheme is denoted by $\gamma(\cdot)$.

For a given probabilistic power allocation $\Gamma(\cdot)$, the average rate, the average power, and the outage probability are, respectively, given by

$$
\begin{aligned}
\mathbb{E}[I(\Gamma(S), S)] & =\iint I(\gamma, s) f_{\Gamma \mid S}(\gamma \mid s) d \gamma d F(s) \\
\mathbb{E}[\Gamma(S)] & =\iint \gamma(s) f_{\Gamma \mid S}(\gamma \mid s) d \gamma d F(s)
\end{aligned}
$$

and

$$
\begin{aligned}
\operatorname{Pr}\{I(\Gamma(S), S) & \left.<r_{0}\right\} \\
= & \iint 1\left(I(\gamma, s)<r_{0}\right) f_{\Gamma \mid S}(\gamma \mid s) d \gamma d F(s)
\end{aligned}
$$

where $I(\cdot, \cdot)$ is as defined in (3), and $1(\cdot)$ denotes the indicator function.

The service-outage-based power and rate allocation problem is stated below. In the following, we assume that $A>0, \lambda_{0} \geq 0$, $0 \leq \sigma \leq 1, r_{0} \geq 0$, and $0 \leq \epsilon \leq 1$ are fixed.

Problem 1: Determine the maximum average information rate

$$
R^{*}=\max _{f_{\Gamma \mid S}} \mathbb{E}[I(\Gamma(S), S)]
$$

subject to the constraints

$$
\begin{aligned}
0 \leq \Gamma(S) & \leq 1, \quad \text { w.p. } 1 \\
\mathbb{E}[\Gamma(S)] & \leq \sigma \\
\operatorname{Pr}\left\{I(\Gamma(S), S)<r_{0}\right\} & \leq \epsilon
\end{aligned}
$$

where the conditional pdf $f_{\Gamma \mid S}$ is a set of functions satisfying

$$
\int f_{\Gamma \mid S}(\gamma \mid s) d \gamma=1, \quad f_{\Gamma \mid S}(\gamma \mid s) \geq 0 \text { for every } s .
$$

The maximum average rate $R^{*}$ in $(8)$ is called the serviceoutage-based achievable rate.

\section{OPtimal POWER Allocation Scheme}

In this section, the solution of Problem 1 is derived in a systematic manner. We begin by introducing two deterministic power allocation schemes which will later turn out to be the components of the optimal solution. These deterministic schemes are themselves solutions of two well-known problems. The first scheme is the solution of the ergodic capacity problem (introduced as Problem 2). The second scheme, which solves the basic rate allocation problem (introduced as Problem 3) is intimately related to the outage capacity problem (introduced as Problem 4). After outlining these deterministic schemes, we discuss the conditions under which Problem 1 has a feasible solution. Finally, the solution of Problem 1 is derived in a sequence of steps. The basic idea is to show that the optimal solution is a two-layer power allocation problem, where the first layer comprises the ergodic capacity power allocation, and the 
second layer comprises an additional level of power allocation for the states in which the first layer cannot guarantee the basic rate requirement. Our proof technique is very similar to [15] in which the same problem was solved for the RF fading channel.

In stating our results, it is convenient to use the following notation:

$$
\xi(x)=\frac{1}{x}\left(e^{-1}(1+x)^{(1+1 / x)}-1\right), \quad x \geq 0
$$

whence from (4) it can be verified that

$$
\begin{aligned}
\zeta(x, y) & -x(1+\log y) \\
= & x \log \left(1+\xi\left(\frac{x}{y}\right) \frac{x}{y}\right), \quad x \geq 0, \quad y \geq 0
\end{aligned}
$$

and from (3), it readily follows that

$$
I(\gamma, s)=\gamma s A \log \left(\frac{1+\frac{s A}{\lambda_{0}} \xi\left(\frac{s A}{\lambda_{0}}\right)}{1+\frac{\gamma s A}{\lambda_{0}} \xi\left(\frac{\gamma s A}{\lambda_{0}}\right)}\right) .
$$

\section{A. Deterministic Power Allocation Schemes}

We introduce two deterministic power allocation schemes, which will later be used to characterize the solution of Problem 1. The first scheme corresponds to the optimal solution of the ergodic capacity problem, and the second scheme corresponds to the basic rate power allocation that requires the minimum average power to maintain a basic rate at each channel fade.

Consider first the ergodic capacity problem, which is stated in Problem 2. Its solution is a power allocation scheme that maximizes the average mutual information subject to the peak and average power constraints.

Problem 2: Determine the maximum average information rate

$$
C_{\mathrm{erg}}(\sigma)=\max _{\gamma: \mathbb{R}^{+} \rightarrow[0,1]} \mathbb{E}[I(\gamma(S), S)]
$$

subject to the average power constraint $\mathbb{E}[\gamma(S)] \leq \sigma$.

The solution of Problem 2 has been outlined in [2, Corollary 2] and is stated here for the sake of completeness. The optimal power allocation scheme $\gamma_{\text {erg }}^{*}: \mathbb{R}^{+} \rightarrow[0,1]$ that achieves the maximum in (16) is given as follows. For $\rho \geq 0, s \in \mathbb{R}^{+}$, let

$$
\gamma_{\rho}(s) \triangleq \frac{\lambda_{0}}{s A}\left(e^{-(1+\rho / s A)}\left(1+\frac{s A}{\lambda_{0}}\right)^{\left(1+\lambda_{0} / s A\right)}-1\right)
$$

and let

$$
\sigma_{0} \triangleq \mathbb{E}\left[\gamma_{0}(S)\right]
$$

If $\sigma_{0}>\sigma$, let $\rho=\rho^{*}>0$ be the solution of the equation $\mathbb{E}\left[\left[\gamma_{\rho}(S)\right]^{+}\right]=\sigma$, where we have used the notation $[x]^{+}=$ $\max \{0, x\}$. Then, the optimal power allocation scheme $\gamma_{\text {erg }}^{*}$ in (16) is given by

$$
\gamma_{\text {erg }}^{*}(s)=\left\{\begin{array}{ll}
\gamma_{0}(s), & \sigma \geq \sigma_{0} \\
{\left[\gamma_{\rho^{*}}(s)\right]^{+},} & \sigma<\sigma_{0},
\end{array} \quad s \in \mathbb{R}^{+} .\right.
$$

Consider next the basic rate allocation problem, which is stated in Problem 3. The solution of the basic rate allocation problem is the minimum average power required to satisfy a given basic rate requirement. Note that the basic rate allocation problem is stated for a specific channel state, and the basic rate requirement is imposed on the instantaneous mutual information.

Problem 3: For fixed $r_{0}>0$ and $s \in \mathbb{R}^{+}$, determine the minimum average power

$$
\gamma_{\text {basic }}^{*}\left(s, r_{0}\right)=\min _{0 \leq \gamma \leq 1} \gamma
$$

subject to the basic rate requirement $I(\gamma, s)=r_{0}$.

Problem 3 does not necessarily have a solution for an arbitrary choice of $r_{0}$ and $s$. This follows from the fact that for the Poisson fading channel, the instantaneous mutual information is a concave ${ }^{3}$ but not a monotonically increasing function of the transmitter power (measured in terms of the duty cycle), as opposed to the RF fading channel. See Fig. 2. For a fixed channel fade $s \in \mathbb{R}^{+}$, it can be shown that the maximum instantaneous mutual information is achieved with conditional duty cycle $^{4} \gamma_{0}(s)=\xi\left(s A / \lambda_{0}\right)$, and is given by

$$
\begin{aligned}
& I_{\max }(s) \\
& \quad \triangleq I\left(\gamma_{0}(s), s\right) \\
& \quad=\lambda_{0}\left[\frac{s A}{\lambda_{0}} \xi\left(\frac{s A}{\lambda_{0}}\right)-\log \left(1+\frac{s A}{\lambda_{0}} \xi\left(\frac{s A}{\lambda_{0}}\right)\right)\right] \\
& \quad=\lambda_{0}\left[e^{-1}\left(1+\frac{s A}{\lambda_{0}}\right)^{\left(1+\lambda_{0} / s A\right)}-\left(1+\frac{\lambda_{0}}{s A}\right) \log \left(1+\frac{s A}{\lambda_{0}}\right)\right] .
\end{aligned}
$$

Note that

$$
\begin{aligned}
\frac{d I_{\max }}{d s}= & \frac{\lambda_{0}^{2}}{s^{2} A}\left(\frac{s A}{\lambda_{0}}-\log \left(1+\frac{s A}{\lambda_{0}}\right)\right) \\
& \times\left(e^{-1}\left(1+\frac{s A}{\lambda_{0}}\right)^{\left(1+\lambda_{0} / s A\right)}-1\right) \\
= & \frac{\lambda_{0}}{s}\left(\frac{s A}{\lambda_{0}}-\log \left(1+\frac{s A}{\lambda_{0}}\right)\right) \xi\left(\frac{s A}{\lambda_{0}}\right) \\
\geq & 0, \quad s \in \mathbb{R}^{+}
\end{aligned}
$$

since $x-\log (1+x)$ is nonnegative for $x \geq 0$ and $\xi(x)$ is monotone decreasing for $x \geq 0$ with $\xi(0)=1 / 2$ and $\xi(\infty)=$ $1 / e$ (cf., e.g., [5, Fig. 2]). Therefore, $I_{\max }(s)$ is a monotonically increasing function of $s \in \mathbb{R}^{+}$.

By the concavity of mutual information, it follows that for a basic rate $r_{0} \leq I_{\max }(s)$, there are two real solutions of the equation $I(\gamma, s)=r_{0}$; see Fig. 2. Let these two real solutions be denoted by $\gamma_{L}\left(s, r_{0}\right)$ and $\gamma_{H}\left(s, r_{0}\right)$, where $\gamma_{L}\left(s, r_{0}\right) \leq$ $\gamma_{0}(s) \leq \gamma_{H}\left(s, r_{0}\right)$ with equality if and only if $r_{0}=I_{\max }(s)$.

We now consider a numerical example that illustrates typical values of the various parameters defined above.

Example 1: Assume $A=10, \lambda_{0}=1$, and consider a fixed fade level $s=1$. In Fig. 2, the instantaneous mutual information $I(\gamma, s)$ versus transmitter duty cycle $\gamma$ is plotted for $s=1$.

${ }^{3}$ The concavity of instantaneous mutual information can be seen from the fact that $\partial^{2} I / \partial \gamma^{2}=-s^{2} A^{2} /\left(\gamma s A+\lambda_{0}\right) \leq 0$.

${ }^{4}$ This result can be readily inferred from [5, Th. 1]. In the notation of [5], $\gamma_{0}(s)=k_{m} / c$ with $c=s A$. 


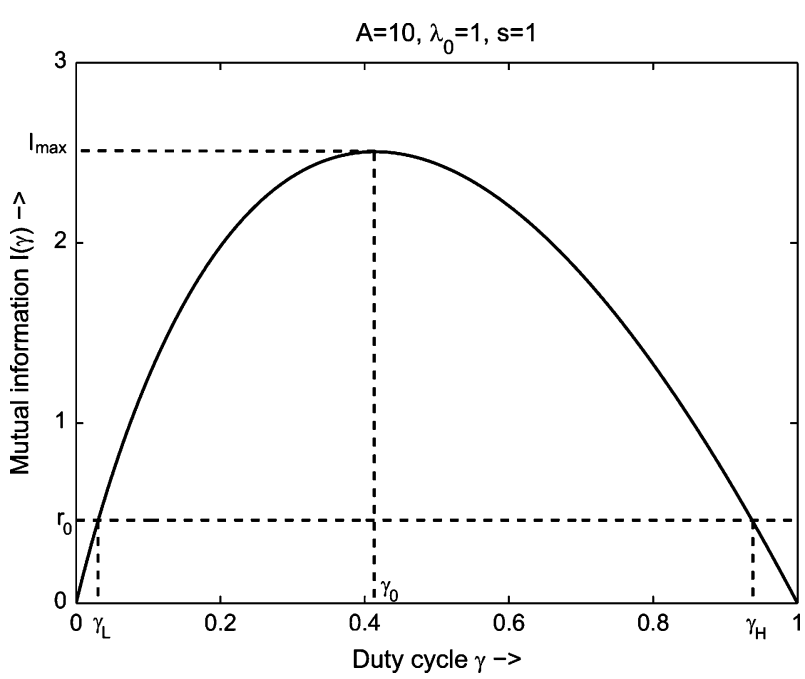

Fig. 2. Plot of instantaneous mutual information versus transmitter duty cycle for a given channel fade. In this example, we set $A=10, \lambda_{0}=1$, and $s=1$. The maximum mutual information $I_{\max }(s)=2.5056$ nats/unit time is obtained for the duty cycle $\gamma_{0}(s)=0.415$. The basic rate $r_{0}=0.461$ nats/unit time is satisfied for the duty cycles $\gamma_{L}\left(s, r_{0}\right)=0.031$ and $\gamma_{H}\left(s, r_{0}\right)=0.937$.

The mutual information first increases with $\gamma$ until it reaches the maximum $I_{\max }(s)=2.5056$ nats/unit time when $\gamma=\gamma_{0}(s)=$ 0.415 , and then decreases for higher values of $\gamma$. For a basic rate $r_{0}=0.461$ nats/unit time, the two solutions of $I(\gamma, s)=r_{0}$ are $\gamma_{L}\left(s, r_{0}\right)=0.031$ and $\gamma_{H}\left(s, r_{0}\right)=0.937$, respectively.

We emphasize that Problem 3 may not necessarily have a solution for any arbitrary choice of $r_{0}>0$ and $s \in \mathbb{R}^{+}$. In fact, for a fixed basic rate $r_{0}>0$, the set

$$
\mathcal{S}_{0}\left(r_{0}\right) \triangleq\left\{s \in \mathbb{R}^{+}: I_{\max }(s)<r_{0}\right\}
$$

comprises channel fades for which the instantaneous mutual information will always remain below the basic rate, irrespective of the choice of the duty cycle $\gamma \in[0,1]$. The set $\mathcal{S}_{0}\left(r_{0}\right)$ is defined as the outage floor set for the basic rate $r_{0}$, and this set constitutes of channel states for which Problem 3 is not solvable. This property of the Poisson fading channel is markedly different from the RF fading channel, where a solution always exists for the corresponding basic rate allocation problem [15]. Furthermore, by the monotonicity of $I_{\max }(\cdot)$, it follows that the outage floor set is of the form $\mathcal{S}_{0}\left(r_{0}\right)=\left[0, s_{0}\left(r_{0}\right)\right)$ where $s_{0}\left(r_{0}\right)=I_{\max }^{-1}\left(r_{0}\right)$ is the smallest value of channel fade for which the basic rate $r_{0}$ can be supported. For future reference, define

$$
P_{0}\left(r_{0}\right) \triangleq \int_{\mathcal{S}_{0}\left(r_{0}\right)} d F(s)
$$

as the probability of the outage floor set $\mathcal{S}_{0}\left(r_{0}\right)$. It follows that Problem 3 has a solution only if $s \in \mathcal{S}_{0}^{c}\left(r_{0}\right)=\left[s_{0}\left(r_{0}\right), \infty\right)$, and the solution, when it exists, is given by

$$
\gamma_{\text {basic }}^{*}\left(s, r_{0}\right)=\gamma_{L}\left(s, r_{0}\right)
$$

where $\gamma_{L}(\cdot)$ is the smallest real solution to $I(\gamma, s)=r_{0}$.

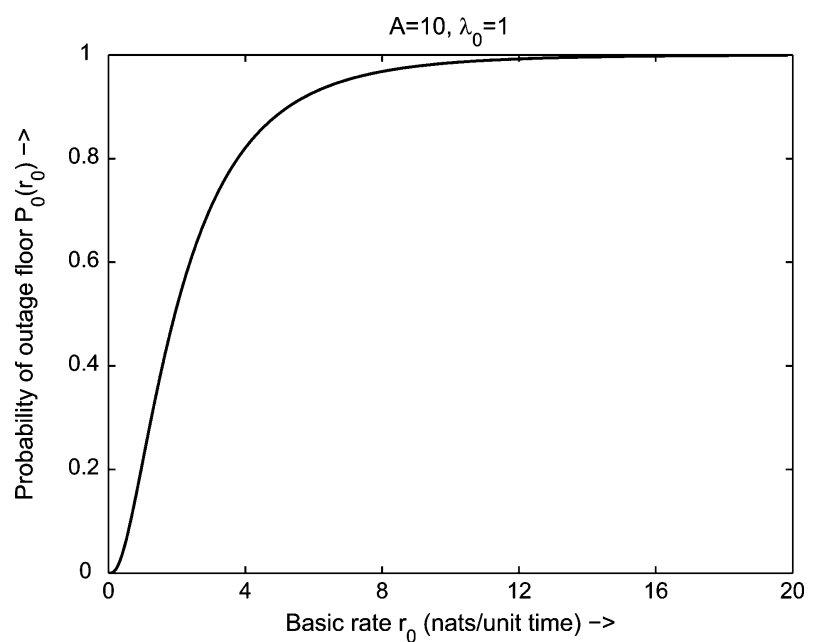

Fig. 3. The probability of the outage floor set $P_{0}\left(r_{0}\right)$ is plotted against the basic rate requirement $r_{0}$ for the lognormal fading channel in Example 2.

Example 2: In this example and all the subsequent examples in this paper, we will consider a Poisson channel with lognormal fading, i.e., $1 / 2 \log S \sim \mathcal{N}\left(\mu_{G}, \sigma_{G}^{2}\right)$, where we assume that $\sigma_{G}^{2}=-\mu_{G}=0.1$, such that the fade is normalized (i.e., $\mathbb{E}[S]=$ $1)$. We also set $A=10, \lambda_{0}=1$. In Fig. 3, the probability of the outage floor set is plotted versus the basic rate. In particular, when the basic rate is $r_{0}=0.461$ nats/unit time, the probability of the outage floor set is $P_{0}\left(r_{0}\right)=0.05$. The minimum fade level for which Problem 3 has a solution is $s_{0}\left(r_{0}\right)=0.2888$.

Example 3: Consider the lognormal fading channel from Example 2. It can readily be verified that for this channel $\sigma_{0}=$ $\mathbb{E}\left[\gamma_{0}(S)\right]=0.42$. For different values of $\sigma$ (viz., $\sigma=0.1,0.25$, and $\sigma \geq \sigma_{0}=0.42$ ), the ergodic capacity-achieving power allocation $\left[\gamma_{\mathrm{erg}}^{*}(\cdot)\right]$ is plotted in Fig. 4(a). The basic rate power allocation corresponding to $r_{0}=0.461$ nats/unit time is also plotted for comparison. In Fig. 4(b), the instantaneous mutual information corresponding to the ergodic capacity-achieving and basic rate power allocations are plotted versus channel fade. Note that the basic rate allocation is active for all fade levels $s \geq s_{0}\left(r_{0}\right)=$ 0.2888 . From Fig. 4(a), we see that although the basic rate power allocation $\gamma_{\text {basic }}^{*}\left(\cdot, r_{0}\right)$ is a monotonically decreasing function of channel state [in the set $\mathcal{S}_{0}^{c}\left(r_{0}\right)$ ], the ergodic capacity power allocation $\gamma_{\mathrm{erg}}^{*}(\cdot)$ is not necessarily monotone with respect to channel fade. In fact, $\gamma_{\mathrm{erg}}^{*}(\cdot)$ is monotone increasing for small values of $\sigma$ and monotone decreasing for $\sigma \geq \sigma_{0}$. For intermediate values of $\sigma, \gamma_{\mathrm{erg}}^{*}(\cdot)$ first increases and then decreases with $s$. This behavior of the ergodic capacity-achieving power allocation scheme for the Poisson fading channel is markedly different from the RF fading channel, where the ergodic capacity power allocation (popularly known as waterfilling [6]) is monotone increasing with respect to channel fade. However, for a fixed channel fade $s \in \mathbb{R}^{+}$, the instantaneous mutual information $I(\gamma, s)$ is a monotone function of power allocation $\gamma$ for $\gamma \leq \gamma_{0}(s)$ (see Fig. 2), which leads to the following result.

Proposition 1: For any $s \in \mathcal{S}_{0}^{c}\left(r_{0}\right)$, we have

$$
I\left(\gamma_{\text {erg }}^{*}(s), s\right) \geq r_{0} \quad \Longleftrightarrow \quad \gamma_{\text {erg }}^{*}(s) \geq \gamma_{\text {basic }}^{*}\left(s, r_{0}\right)
$$




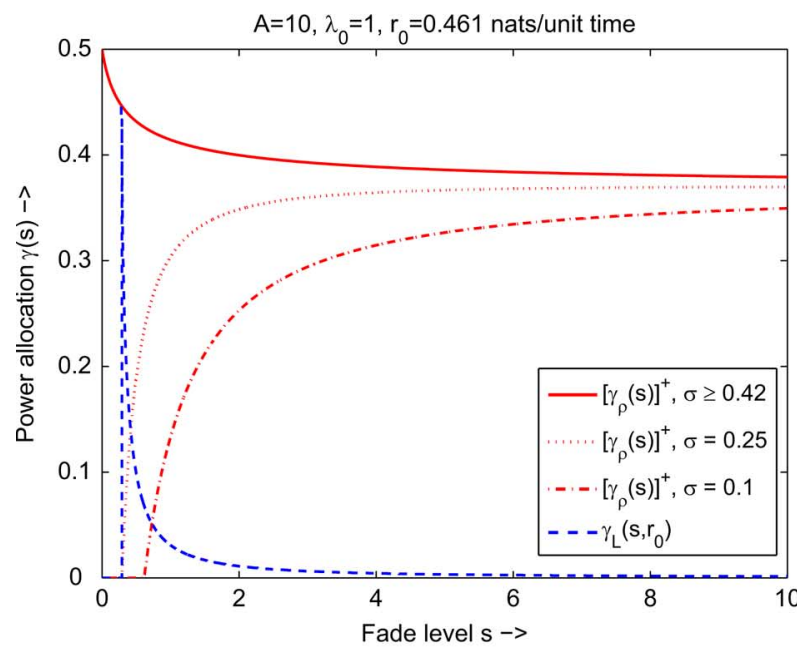

(a) Power allocation

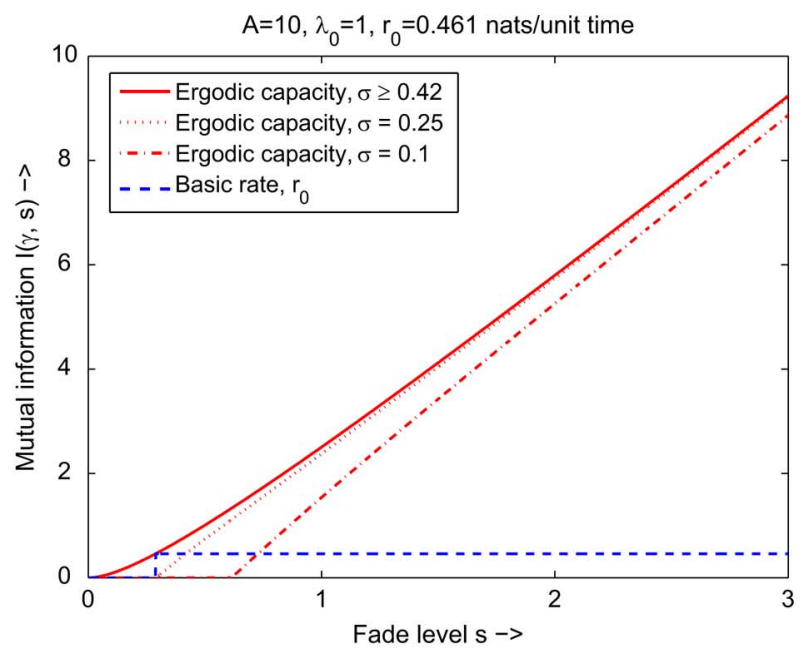

(b) Instantaneous mutual information

Fig. 4. Power allocation and instantaneous mutual information versus channel fade corresponding to both ergodic capacity and basic rate power allocations for the lognormal fading channel in Example 3. The basic rate is fixed at $r_{0}=0.461$ nats/unit time. Ergodic capacity power allocations are shown for different values of $\sigma$. The basic rate power allocation is active for fades $s \geq s_{0}\left(r_{0}\right)=0.2888$.

Proof: From (19) and (24), it follows that $\gamma_{\text {erg }}^{*}(s) \leq \gamma_{0}(s)$ and $\gamma_{\text {basic }}^{*}\left(s, r_{0}\right) \leq \gamma_{0}(s)$, respectively. Furthermore, by definition, $I\left(\gamma_{\text {basic }}^{*}(s), s\right)=r_{0}$. Therefore, it suffices to prove that for a fixed $s \in \mathbb{R}^{+}, I(\gamma, s)$ is monotone increasing for $\gamma \leq \gamma_{0}(s)$. From (3) and (14), it follows that

$$
\begin{aligned}
\frac{\partial I}{\partial \gamma} & =\zeta\left(s A, \lambda_{0}\right)-s A\left(1+\log \left(\lambda_{0}+\gamma s A\right)\right) \\
& =s A \log \left(\frac{1+\xi\left(\frac{s A}{\lambda_{0}}\right) \frac{s A}{\lambda_{0}}}{1+\gamma \frac{s A}{\lambda_{0}}}\right) .
\end{aligned}
$$

Clearly, $\partial I / \partial \gamma \geq 0$ for $\gamma \leq \gamma_{0}(s)=\xi\left(s A / \lambda_{0}\right)$. This completes the proof.

The basic rate power allocation $\gamma_{\text {basic }}^{*}(\cdot)$ discussed above is intimately connected to the outage capacity problem for Poisson fading channel [4]. The outage capacity $C_{\text {out }}(\sigma, \epsilon)$ is the maximum basic rate achievable with a probability of outage constraint $\epsilon$ and the peak and average power constraints $A$ and $\sigma$, respectively. Mathematically

$$
C_{\text {out }}(\sigma, \epsilon) \triangleq \max \left\{R \geq 0: P_{\text {out }}(R, \sigma) \leq \epsilon\right\}
$$

where the minimum probability of outage $P_{\text {out }}$ is the solution to the capacity versus outage probability problem defined in Problem 4 . The outage capacity is achieved by the power allocation that has $\epsilon$ as the minimum outage probability subject to the peak and average power constraints $A$ and $\sigma$, respectively. The solution of Problem 4 requires, as a vital ingredient, the basic rate power allocation scheme that solved Problem 3.

Problem 4: Given a basic rate $r_{0}>0$, determine the minimum probability of outage

$$
P_{\text {out }}\left(r_{0}, \sigma\right)=\min _{\gamma: \mathbb{R}^{+} \rightarrow[0,1]} \operatorname{Pr}\left(I(\gamma(S), S)<r_{0}\right)
$$

subject to the average power constraint $\mathbb{E}[\gamma(S)] \leq \sigma$.
The optimal power allocation scheme that achieves the minimum outage probability in (28) can be characterized in terms of the basic rate power allocation (24) and the average power constraint $\sigma$. This is a special case of [4, Th. 1], corresponding to $N=1$ transmit aperture. For the sake of completeness, the solution is discussed below. For $p \in[0,1]$, define the sets

$$
\begin{aligned}
& \mathcal{R}(p)=\left\{s \in \mathcal{S}_{0}^{c}\left(r_{0}\right): \gamma_{\text {basic }}^{*}\left(s, r_{0}\right)<p\right\} \\
& \overline{\mathcal{R}}(p)=\left\{s \in \mathcal{S}_{0}^{c}\left(r_{0}\right): \gamma_{\text {basic }}^{*}\left(s, r_{0}\right) \leq p\right\}
\end{aligned}
$$

and the boundary set of $\mathcal{R}(p)$ as

$$
\mathcal{B}(p)=\left\{s \in \mathcal{S}_{0}^{c}\left(r_{0}\right): \gamma_{\text {basic }}^{*}\left(s, r_{0}\right)=p\right\} .
$$

Furthermore, define

$$
\begin{aligned}
& \Sigma(p)=\int_{\mathcal{R}(p)} \gamma_{\text {basic }}^{*}\left(s, r_{0}\right) d F(s) \\
& \bar{\Sigma}(p)=\int_{\overline{\mathcal{R}}(p)} \gamma_{\text {basic }}^{*}\left(s, r_{0}\right) d F(s) .
\end{aligned}
$$

In order to state the optimal power allocation scheme for Problem 4 , we also define the duty cycle threshold $p^{*}$ and weight $w^{*}$, respectively, as

$$
\begin{aligned}
p^{*} & =\sup \{p: \Sigma(p)<\sigma\} \\
w^{*} & =\frac{\sigma-\Sigma\left(p^{*}\right)}{\bar{\Sigma}\left(p^{*}\right)-\Sigma\left(p^{*}\right)} .
\end{aligned}
$$

The optimal power allocation scheme $\gamma_{\text {out }}^{*}: \mathbb{R}^{+} \rightarrow[0,1]$ that solves Problem 4 is given as follows. If $\sigma \geq \mathbb{E}\left[\gamma_{\text {basic }}^{*}\left(S, r_{0}\right)\right]$, then

$$
\gamma_{\text {out }}^{*}(s)= \begin{cases}\gamma_{\text {basic }}^{*}\left(s, r_{0}\right), & \text { if } s \in \mathcal{S}_{0}^{c}\left(r_{0}\right) \\ 0, & \text { if } s \in \mathcal{S}_{0}\left(r_{0}\right) .\end{cases}
$$




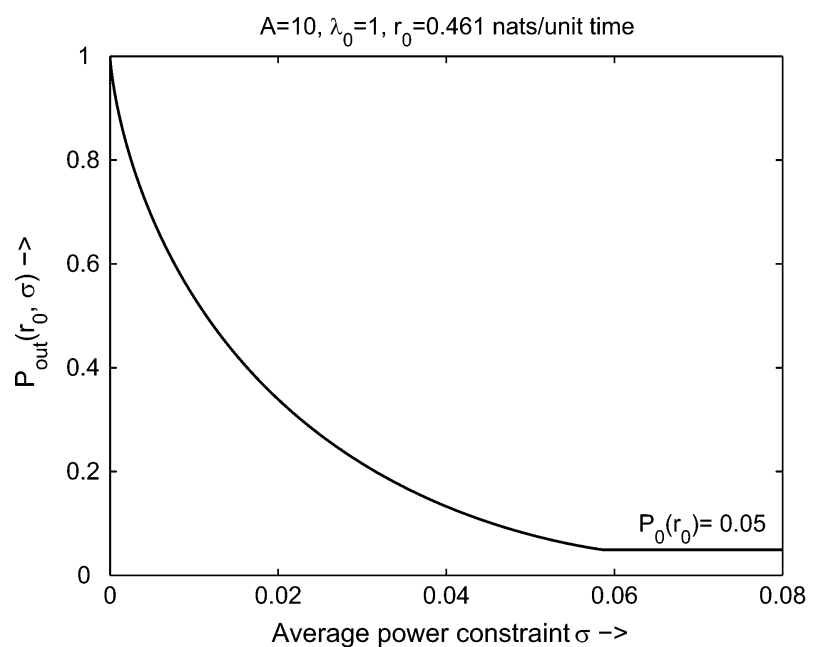

(a) Outage probability

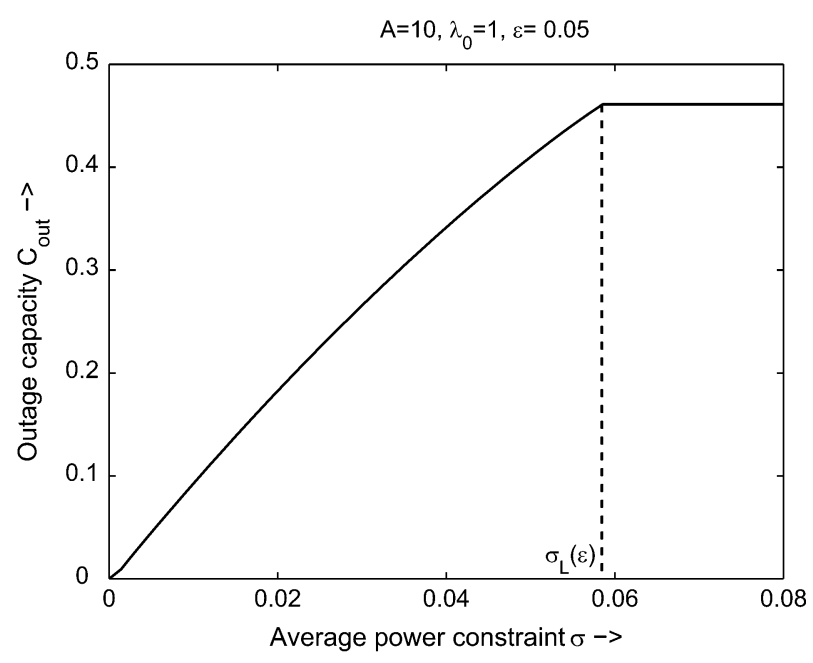

(b) Outage capacity

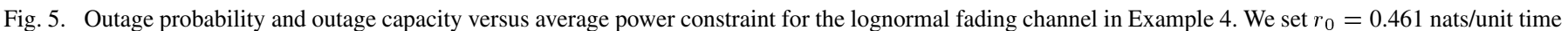

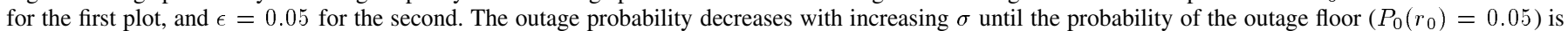

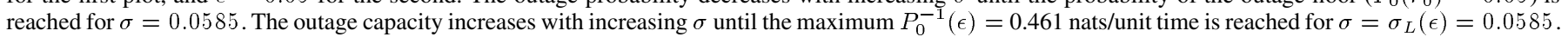

On the other hand, if $\sigma<\mathbb{E}\left[\gamma_{\text {basic }}^{*}\left(S, r_{0}\right)\right]$, then

$$
\gamma_{\text {out }}^{*}(s)= \begin{cases}\gamma_{\text {basic }}^{*}\left(s, r_{0}\right), & \text { if } s \in \mathcal{R}\left(p^{*}\right) \\ 0, & \text { if } s \notin \overline{\mathcal{R}}\left(p^{*}\right)\end{cases}
$$

and if $s \in \mathcal{B}\left(p^{*}\right)$, then $\gamma_{\text {out }}^{*}(s)=\gamma_{\text {basic }}^{*}\left(s, r_{0}\right)$ with probability $w^{*}$ and $\gamma_{\text {out }}^{*}(s)=0$ with probability $1-w^{*}$. Note that for a continuous fading distribution, and hence, for a continuous distribution of $\gamma_{\text {basic }}^{*}$, the set $\mathcal{B}(p)$ has measure zero. Therefore, the randomization with weight $w^{*}$ is not necessary for a continuous fading distribution. Note also that the sets $\overline{\mathcal{R}}\left(p^{*}\right)$ and $\mathcal{S}_{0}^{c}\left(r_{0}\right)$ coincide when $\sigma=\mathbb{E}\left[\gamma_{\text {basic }}^{*}\left(S, r_{0}\right)\right]$.

The minimum outage probability is given by

$$
\begin{aligned}
P_{\text {out }}\left(r_{0}, \sigma\right) & =1-\int_{\overline{\mathcal{R}}\left(p^{*}\right)} d F(s) \\
& =1-w^{*} \operatorname{Pr}\left(S \in \mathcal{B}\left(p^{*}\right)\right)-\operatorname{Pr}\left(S \in \mathcal{R}\left(p^{*}\right)\right) .
\end{aligned}
$$

Recall that the basic rate $r_{0}$ is not achievable for channel fades in the outage floor set $\mathcal{S}_{0}\left(r_{0}\right)$, regardless of the average power constraint $\sigma$. In other words, irrespective of $\sigma$, we must have $P_{\text {out }}\left(r_{0}, \sigma\right) \geq P_{0}\left(r_{0}\right)$, where $P_{0}\left(r_{0}\right)$ is defined in (23) as the probability of the outage floor set $\mathcal{S}_{0}\left(r_{0}\right)$. This implies that for a fixed outage probability $\epsilon$, the outage capacity $C_{\text {out }}(\sigma, \epsilon)$ cannot exceed $P_{0}^{-1}(\epsilon)$, irrespective of $\sigma$. Let $\sigma_{L}(\epsilon) \triangleq \mathbb{E}\left[\gamma_{\text {basic }}^{*}\left(S, P_{0}^{-1}(\epsilon)\right)\right]$ be the minimum average power required to achieve the maximum outage capacity $P_{0}^{-1}(\epsilon)$. It is easy to see that for $\sigma \geq \sigma_{L}(\epsilon), C_{\text {out }}(\sigma, \epsilon)=P_{0}^{-1}(\epsilon)$.

Example 4: Consider again the lognormal channel from Example 2. In Fig. 5(a), we plot the variation of the outage probability $P_{\text {out }}\left(r_{0}, \sigma\right)$ versus $\sigma$ for a fixed basic rate $r_{0}=0.461$ nats/ unit time. As expected, the outage probability decreases with increasing $\sigma$ until the probability of the outage floor $P_{0}\left(r_{0}\right)=$ 0.05 is reached for $\sigma=0.0585$. In Fig. 5(b), we plot the outage capacity $C_{\text {out }}(\sigma, \epsilon)$ versus $\sigma$ for a fixed outage probability $\epsilon=$
0.05. The outage capacity increases with increasing $\sigma$ until the maximum $P_{0}^{-1}(\epsilon)=0.461$ nats/unit time is reached for $\sigma=$ $\sigma_{L}(\epsilon)=0.0585$.

\section{B. Feasibility Issues}

Problem 1 may not be feasible for an arbitrary choice of the parameters $\left(\epsilon, \sigma, r_{0}\right)$. First, in order to satisfy the outage probability constraint (11), the probability of the outage floor set must not exceed $\epsilon$, i.e.,

$$
\epsilon \geq P_{0}\left(r_{0}\right)=\int_{\mathcal{S}_{0}\left(r_{0}\right)} d F(s) .
$$

Moreover, even when (38) is satisfied, the feasibility of Problem 1 is directly related to the outage capacity $C_{\text {out }}$. Clearly, for fixed $(\sigma, \epsilon)$, Problem 1 is feasible if and only if the basic rate requirement does not exceed the outage capacity, i.e., $r_{0} \leq C_{\text {out }}(\sigma, \epsilon)$. Equivalently, we must have

$$
\sigma \geq \sigma_{\min }\left(r_{0}, \epsilon\right)
$$

where $\sigma_{\min }\left(r_{0}, \epsilon\right)$ denotes the minimum average power required in order to support the basic rate $r_{0}$ with an outage probability $\epsilon$. Furthermore, when $\sigma=\sigma_{\min }\left(r_{0}, \epsilon\right)$, i.e., $r_{0}=C_{\text {out }}(\sigma, \epsilon)$, the optimal power allocation scheme that solves Problem 1 must also solve Problem 4.

Remark: It should be pointed out that $\mathbb{E}\left[\gamma_{\text {basic }}^{*}\left(S, r_{0}\right)\right]=$ $\sigma_{\min }\left(r_{0}, \epsilon\right)$ when $\epsilon=P_{0}\left(r_{0}\right)$, implying that $\sigma_{\min }\left(r_{0}, P_{0}\left(r_{0}\right)\right)$ is the minimum power necessary to support the basic rate $r_{0}$ in the entire outage floor complementary set $\mathcal{S}_{0}^{c}\left(r_{0}\right)$.

Example 5: Recall again the lognormal channel in Example 2. For a basic rate $r_{0}=0.461$ nats/unit time, we must have $\epsilon \geq P_{0}\left(r_{0}\right)=0.05$ to ensure feasibility. Additionally, with $\epsilon=0.05$, we need $\sigma \geq \sigma_{\min }=0.0585$ for Problem 1 to have a feasible solution. 


\section{Derivation of the Optimal Probabilistic Power Allocation Scheme}

In this section, we derive the optimal solution of Problem 1 when a solution exists. We separate two cases according to the value of $\sigma$.

Case 1: $\sigma \geq \sigma_{0}$, where $\sigma_{0}$ is as defined in (18). In this case, when (38) is satisfied, it is easy to see that the deterministic power allocation scheme $\gamma_{0}(\cdot)$, which yields the maximum achievable rate $\mathbb{E}\left[I_{\max }(S)\right]$, is the optimal solution of Problem 1 . This can be proved by verifying that $\gamma_{0}(\cdot)$ is a feasible power allocation scheme. Note that

$$
\mathbb{E}\left[\gamma_{0}(S)\right]=\sigma_{0} \leq \sigma
$$

so that the average power constraint (10) is satisfied, and

$$
\begin{aligned}
\operatorname{Pr}\left\{I\left(\gamma_{0}(S), S\right)<r_{0}\right\} & =\operatorname{Pr}\left\{S \in \mathcal{S}_{0}\left(r_{0}\right)\right\} \\
& =P_{0}\left(r_{0}\right) \\
& \leq \epsilon
\end{aligned}
$$

by (38), so that the outage constraint (11) is also satisfied. In this case, the service-outage-based achievable rate is given by

$$
R^{*}=\mathbb{E}\left[I_{\max }(S)\right]
$$

which is also the ergodic capacity when $\sigma \geq \sigma_{0}$.

Case 2: $\sigma<\sigma_{0}$. In this case, $\gamma_{0}(\cdot)$ is not a feasible power allocation scheme, since it does not satisfy the average power constraint (10). The first step is to demonstrate that the optimal power allocation scheme is a randomization between two deterministic schemes. By concavity of the mutual information $I(\cdot, \cdot)$, we have the following result.

Proposition 2: Suppose $\sigma<\sigma_{0}$, and let the feasibility conditions (38) and (39) hold. Then, there exists an optimal solution of Problem 1 of the form

$$
\Gamma^{*}(s)= \begin{cases}\gamma_{a}(s), & \text { w. p. } w(s) \\ \gamma_{b}(s), & \text { w. p. } 1-w(s)\end{cases}
$$

where the timesharing factor $w(s)=0$ for all $s \in \mathcal{S}_{0}\left(r_{0}\right)$, the rate function $I\left(\gamma_{a}(s), s\right) \geq r_{0}$ for all $s \in \mathcal{S}_{0}^{c}\left(r_{0}\right), \mathbb{E}[w(S)] \geq$ $1-\epsilon$, and $\mathbb{E}\left[\Gamma^{*}(S)\right]=\sigma$

Proof: See Appendix I.

By Proposition 2, clearly the average power constraint (10) is also satisfied. Furthermore, the conditions $w(s)=0$ for all $s \in \mathcal{S}_{0}\left(r_{0}\right), I\left(\gamma_{a}(s), s\right) \geq r_{0}$ for all $s \in \mathcal{S}_{0}^{c}\left(r_{0}\right)$ and $\mathbb{E}[w(S)] \geq 1-\epsilon$ guarantee that the service outage constraint (10) is satisfied [see (73) in Appendix I]. Thus, Problem 1 reduces to the following optimization problem when $\sigma<\sigma_{0}$.

Problem 5: Determine the maximum expected rate

$$
\max _{\gamma_{a}, \gamma_{b}, w} \mathbb{E}\left[w(S) I\left(\gamma_{a}(S), S\right)+(1-w(S)) I\left(\gamma_{b}(S), S\right)\right]
$$

subject to the constraints

$$
\begin{aligned}
\sigma & =\mathbb{E}\left[w(S) \gamma_{a}(S)+(1-w(S)) \gamma_{b}(S)\right] \\
\mathbb{E}[w(S)] & \geq 1-\epsilon
\end{aligned}
$$

$$
\begin{aligned}
I\left(\gamma_{a}(s), s\right) & \geq r_{0} \quad \forall s \in \mathcal{S}_{0}^{c}\left(r_{0}\right) \\
w(s) & =0 \quad \forall s \in \mathcal{S}_{0}\left(r_{0}\right) \\
0 & \leq \gamma_{a}(s) \leq 1, \quad 0 \leq \gamma_{b}(s) \leq 1, \quad 0 \leq w(s) \leq 1 .
\end{aligned}
$$

By the concavity of $I(\cdot, s)$, it follows that the objective function in (42) is a concave functional of $\left(w(\cdot), \gamma_{a}(\cdot), \gamma_{b}(\cdot)\right)$. The constraints (43)-(47) specify a convex feasible set. Therefore, Problem 5 is a convex functional optimization problem, and the generalized Karush-Kuhn-Tucker (KKT) conditions (cf., e.g., $[15$, Sec. III] for a brief review or [13, Ch. 9] for a detailed exposition) are necessary and sufficient at optimality. In order to characterize the optimal solution of Problem 5, define the Lagrangian

$$
\begin{aligned}
& L\left(s, \gamma_{a}(s), \gamma_{b}(s), w(s), \rho_{1}, \rho_{2}, u_{1}(s)\right) \\
& =w(s) I\left(\gamma_{a}(s), s\right)+(1-w(s)) I\left(\gamma_{b}(s), s\right) \\
& \quad-\rho_{1}\left[w(s) \gamma_{a}(s)+(1-w(s)) \gamma_{b}(s)\right] \\
& \quad+\rho_{2} w(s)+u_{1}(s) I\left(\gamma_{a}(s), s\right)
\end{aligned}
$$

where $\rho_{1}, \rho_{2} \geq 0$, and $u_{1}(\cdot) \geq 0$ are the Lagrange multipliers corresponding to constraints (43)-(45), respectively. Let $\gamma_{a}^{*}(\cdot)$, $\gamma_{b}^{*}(\cdot)$, and $w^{*}(\cdot)$ denote the optimal solution of (42), and $\rho_{1}^{*}, \rho_{2}^{*}$, and $u_{1}^{*}(\cdot)$ denote the optimal values of the respective Lagrange multipliers. The necessary and sufficient KKT conditions ${ }^{5}$ are given by

$$
\begin{aligned}
& \frac{\partial L(\cdots)}{\partial \gamma_{a}^{*}(s)}\left\{\begin{array}{lll}
\leq & 0, & \gamma_{a}^{*}(s)=0 \\
= & 0, & 0<\gamma_{a}^{*}(s)<1 \\
\geq & 0, & \gamma_{a}^{*}(s)=1
\end{array}\right. \\
& \frac{\partial L(\cdots)}{\partial \gamma_{b}^{*}(s)}\left\{\begin{array}{lll}
\leq & 0, & \gamma_{b}^{*}(s)=0 \\
= & 0, & 0<\gamma_{b}^{*}(s)<1 \\
\geq & 0, & \gamma_{b}^{*}(s)=1
\end{array}\right.
\end{aligned}
$$

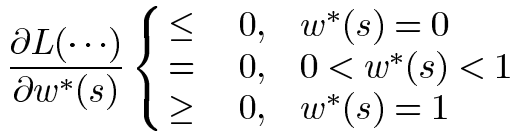

$$
\begin{aligned}
& \sigma=\mathbb{E}\left[w^{*}(S) \gamma_{a}^{*}(S)+\left(1-w^{*}(S)\right) \gamma_{b}^{*}(S)\right], \quad \rho_{1}^{*} \geq 0 \\
& 0=\rho_{2}^{*}\left(\mathbb{E}\left[w^{*}(S)\right]-(1-\epsilon)\right), \quad \rho_{2}^{*} \geq 0 \\
& 0=u_{1}^{*}(s)\left(I\left(\gamma_{a}^{*}(s), s\right)-r_{0}\right), \quad u_{1}^{*}(s) \geq 0 \quad \forall s \in \mathcal{S}_{0}^{c}\left(r_{0}\right) \\
& 0=w^{*}(s) \quad \forall s \in \mathcal{S}_{0}\left(r_{0}\right) .
\end{aligned}
$$

Condition (55) implies that for $s \in \mathcal{S}_{0}\left(r_{0}\right)$, no randomized power allocation scheme is necessary, since $\Gamma^{*}(s)=\gamma_{b}^{*}(s)$ with probability 1 . The deterministic power allocation scheme $\gamma_{a}^{*}(\cdot)$ plays a significant role only for fades $s \in \mathcal{S}_{0}^{c}\left(r_{0}\right)$. Evaluating (49), (50), and (54), we arrive at the following result.

Proposition 3: Suppose $\sigma<\sigma_{0}$. For $s \in \mathcal{S}_{0}^{c}\left(r_{0}\right)$, the optimal $\gamma_{a}^{*}(\cdot)$ is of the form

$$
\gamma_{a}^{*}(s)= \begin{cases}{\left[\gamma_{\rho_{1}^{*}}(s)\right]^{+},} & \text {if } I\left(\left[\gamma_{\rho_{1}^{*}}(s)\right]^{+}, s\right) \geq r_{0} \\ \gamma_{L}\left(s, r_{0}\right), & \text { otherwise }\end{cases}
$$

${ }^{5}$ For brevity, we use the notation $L(\cdots)=L\left(s, \gamma_{a}^{*}(s), \gamma_{b}^{*}(s)\right.$, $\left.w^{*}(s), \rho_{1}^{*}, \rho_{2}^{*}, u_{1}^{*}(s)\right)$, and denote by $\partial L(\cdots) / \partial \gamma_{a}^{*}(s)$ the derivative of $L$ with respect to $\gamma_{a}(s)$ evaluated at $\left(s, \gamma_{a}^{*}(s), \gamma_{b}^{*}(s), w^{*}(s), \rho_{1}^{*}, \rho_{2}^{*}, u_{1}^{*}(s)\right)$, and so on 
and for $s \in \mathbb{R}^{+}$, the optimal $\gamma_{b}^{*}(\cdot)$ is

$$
\gamma_{b}^{*}(s)=\left[\gamma_{\rho_{1}^{*}}(s)\right]^{+} .
$$

\section{Proof: See Appendix II.}

Remark: Proposition 3 implies that the two deterministic power allocation schemes that constitute the basis for the optimal randomized power allocation scheme are: a) the ergodic power allocation $\gamma_{\text {erg }}^{*}(\cdot)$ with parameter $\rho_{1}^{*}$ [see (19)] and b) the basic rate power allocation $\gamma_{\text {basic }}^{*}\left(\cdot, r_{0}\right)$ [see (24)]. In particular, $\gamma_{a}^{*}(\cdot)$ comprises the basic rate power allocation only when the ergodic power allocation scheme cannot meet the basic rate requirement, while $\gamma_{b}^{*}(\cdot)$ is always the ergodic power allocation scheme. The parameter $\rho_{1}^{*}$ and the timesharing factor $w^{*}(\cdot)$ are chosen such that the average power constraint (52) is satisfied.

Define the supplemental power allocation

$$
\begin{aligned}
\gamma_{\text {supp }}(s, \rho) \triangleq\left[\gamma_{L}\left(s, r_{0}\right)-\left[\gamma_{\rho}(s)\right]^{+}\right]^{+}, & \\
s & \in \mathcal{S}_{0}^{c}\left(r_{0}\right), \quad \rho \geq 0
\end{aligned}
$$

which can be interpreted as the additional power required for the ergodic power allocation scheme to meet the basic rate requirement. Note that the supplemental power allocation $\gamma_{\text {supp }}(s, \cdot)$ is defined only for fades $s \in \mathcal{S}_{0}^{c}\left(r_{0}\right)$. By Propositions 1 and 3, an equivalent representation of $\gamma_{a}^{*}(\cdot)$ is

$$
\gamma_{a}^{*}(s)=\left[\gamma_{\rho_{1}^{*}}(s)\right]^{+}+\gamma_{\text {supp }}\left(s, \rho_{1}^{*}\right), \quad s \in \mathcal{S}_{0}^{c}\left(r_{0}\right) .
$$

The instantaneous rate achieved by $\gamma_{a}^{*}(\cdot)$ can similarly be expressed as

$I\left(\gamma_{a}^{*}(s), s\right)=I\left(\left[\gamma_{\rho_{1}^{*}}(s)\right]^{+}, s\right)+I_{\text {supp }}\left(s, \rho_{1}^{*}\right), \quad s \in \mathcal{S}_{0}^{c}\left(r_{0}\right)$

where

$$
I_{\text {supp }}(s, \rho) \triangleq\left[r_{0}-I\left(\left[\gamma_{\rho}(s)\right]^{+}, s\right)\right]^{+}
$$

is the additional rate allocation needed beyond the instantaneous rate achieved by the ergodic power allocation scheme to meet the basic rate requirement.

From Propositions 2 and 3 and the expression (59), we get that the optimal power allocation is

$$
\Gamma^{*}(s)= \begin{cases}{\left[\gamma_{\rho_{1}^{*}}(s)\right]^{+}+\gamma_{\text {supp }}\left(s, \rho_{1}^{*}\right),} & \text { w. p. } w^{*}(s) \\ {\left[\gamma_{\rho_{1}^{*}}(s)\right]^{+},} & \text {w. p. } 1-w^{*}(s)\end{cases}
$$

where $\rho_{1}^{*}$ and $w^{*}(\cdot)$ are yet to be determined. Plugging (57), (59), and (60) in (48), we get at optimality for $s \in \mathcal{S}_{0}^{c}\left(r_{0}\right)$

$$
\begin{aligned}
& L(\cdots)=I\left(\left[\gamma_{\rho_{1}^{*}}(s)\right]^{+}, s\right)-\rho_{1}^{*}\left[\gamma_{\rho_{1}^{*}}(s)\right]^{+} \\
& +w^{*}(s)\left(\rho_{2}^{*}-\Theta_{\text {supp }}\left(s, \rho_{1}^{*}\right)\right)+u_{1}^{*}(s) r_{0}
\end{aligned}
$$

where we have defined the supplemental cost function

$$
\begin{array}{r}
\Theta_{\text {supp }}(s, \rho) \triangleq \rho \gamma_{\text {supp }}(s, \rho)-I_{\text {supp }}(s, \rho), \\
\quad s \in \mathcal{S}_{0}^{c}\left(r_{0}\right), \quad \rho \geq 0
\end{array}
$$

which provides a measure for the cost of allocating the supplemental power in order to meet the basic rate requirement. Some desirable properties of the supplemental cost function are stated below.
Proposition 4: The supplemental cost function $\Theta_{\text {supp }}(\cdot, \cdot)$ has the following properties.

1) If $s \geq s^{\prime}$, then $\Theta_{\text {supp }}(s, \rho) \leq \Theta_{\text {supp }}\left(s^{\prime}, \rho\right)$.

2) If $\gamma_{\text {supp }}(s, \rho)=0$, then $\Theta_{\text {supp }}(s, \rho)=0$. If $\gamma_{\text {supp }}(s, \rho)>$ 0 , then $\Theta_{\text {supp }}(s, \rho)>0$.

Proof: See Appendix III.

Remark: Proposition 4 shows that a higher cost is associated with a poorer channel state. Intuitively, when the channel is in a "bad" state, it is more expensive to support the basic rate requirement; the associated cost of allocating the supplemental power is quantified by the supplemental cost function.

We now obtain a characterization of the optimal timesharing factor $w^{*}(\cdot)$. From $(62)$, we get

$$
\frac{\partial L(\cdots)}{\partial w^{*}(s)}=\rho_{2}^{*}-\Theta_{\text {supp }}\left(s, \rho_{1}^{*}\right)
$$

which, combined with (51), implies that for $s \in \mathcal{S}_{0}^{c}\left(r_{0}\right)$

$$
w^{*}(s)= \begin{cases}1, & \text { if } \Theta_{\text {supp }}\left(s, \rho_{1}^{*}\right)<\rho_{2}^{*} \\ v^{*}(s), & \text { if } \Theta_{\text {supp }}\left(s, \rho_{1}^{*}\right)=\rho_{2}^{*} \\ 0, & \text { if } \Theta_{\text {supp }}\left(s, \rho_{1}^{*}\right)>\rho_{2}^{*}\end{cases}
$$

where $0 \leq v^{*}(s) \leq 1$ needs to be determined. Clearly, the supplemental cost function $\Theta_{\text {supp }}(\cdot)$ determines the value of the optimal timesharing factor $w^{*}(\cdot)$ and indicates whether the supplemental power should be allocated. Note by condition (53) that when $\rho_{2}^{*}>0$, we must have $\mathbb{E}\left[w^{*}(S)\right]=1-\epsilon$. On the other hand, if $\rho_{2}^{*}=0$, we have from (64) that either $\Theta_{\text {supp }}\left(s, \rho_{1}^{*}\right)=0$ or $w^{*}(s)=0$. This implies that $\Gamma^{*}(s)=\left[\gamma_{\rho_{1}^{*}}(s)\right]^{+}$with probability 1, i.e., no supplemental power allocation is required. Consequently, in this case, $0 \leq v^{*}(s) \leq 1$ can be chosen to be any function that meets the requirement $\mathbb{E}\left[w^{*}(S)\right] \geq 1-\epsilon$. Without loss of generality, we choose $v^{*}(\cdot)$ such that $\mathbb{E}\left[w^{*}(S)\right]=1-\epsilon$.

All the results discussed above can be succinctly combined to arrive at the main result below.

Theorem 1: Under the feasibility conditions (38) and (39), an optimal probabilistic power allocation for Problem 1 is as follows. If $\sigma \geq \sigma_{0}$, then

$$
\Gamma^{*}(s)=\gamma_{0}(s) \quad \text { w. p. } 1 .
$$

If $\sigma<\sigma_{0}$, then

$$
\Gamma^{*}(s)= \begin{cases}\gamma_{L}\left(s, r_{0}\right), & \text { w. p. } w^{*}(s) \\ {\left[\gamma_{\rho_{1}^{*}}(s)\right]^{+},} & \text {w. p. } 1-w^{*}(s)\end{cases}
$$

where for $s \in \mathcal{S}_{0}\left(r_{0}\right), w^{*}(s)=0$, and for $s \in \mathcal{S}_{0}^{c}\left(r_{0}\right)$

$$
w^{*}(s)= \begin{cases}1, & \text { if } \Theta_{\text {supp }}\left(s, \rho_{1}^{*}\right)<\rho_{2}^{*} \\ v^{*}(s), & \text { if } \Theta_{\text {supp }}\left(s, \rho_{1}^{*}\right)=\rho_{2}^{*} \\ 0, & \text { if } \Theta_{\text {supp }}\left(s, \rho_{1}^{*}\right)>\rho_{2}^{*}\end{cases}
$$

where $\rho_{1}^{*} \geq 0, \rho_{2}^{*} \geq 0$, and $0 \leq v^{*}(s) \leq 1$ are solutions of

$$
\begin{aligned}
& \mathbb{E}\left[\Gamma^{*}(S)\right]=\sigma \\
& \mathbb{E}\left[w^{*}(S)\right]=1-\epsilon .
\end{aligned}
$$

Remarks: i) The optimal power allocation scheme can be viewed as a two-layer allocation: the first layer is the (ergodic) capacity-achieving power allocation, and the second layer is the supplemental power allocation. The second layer provides the additional power required for the ergodic power allocation to 

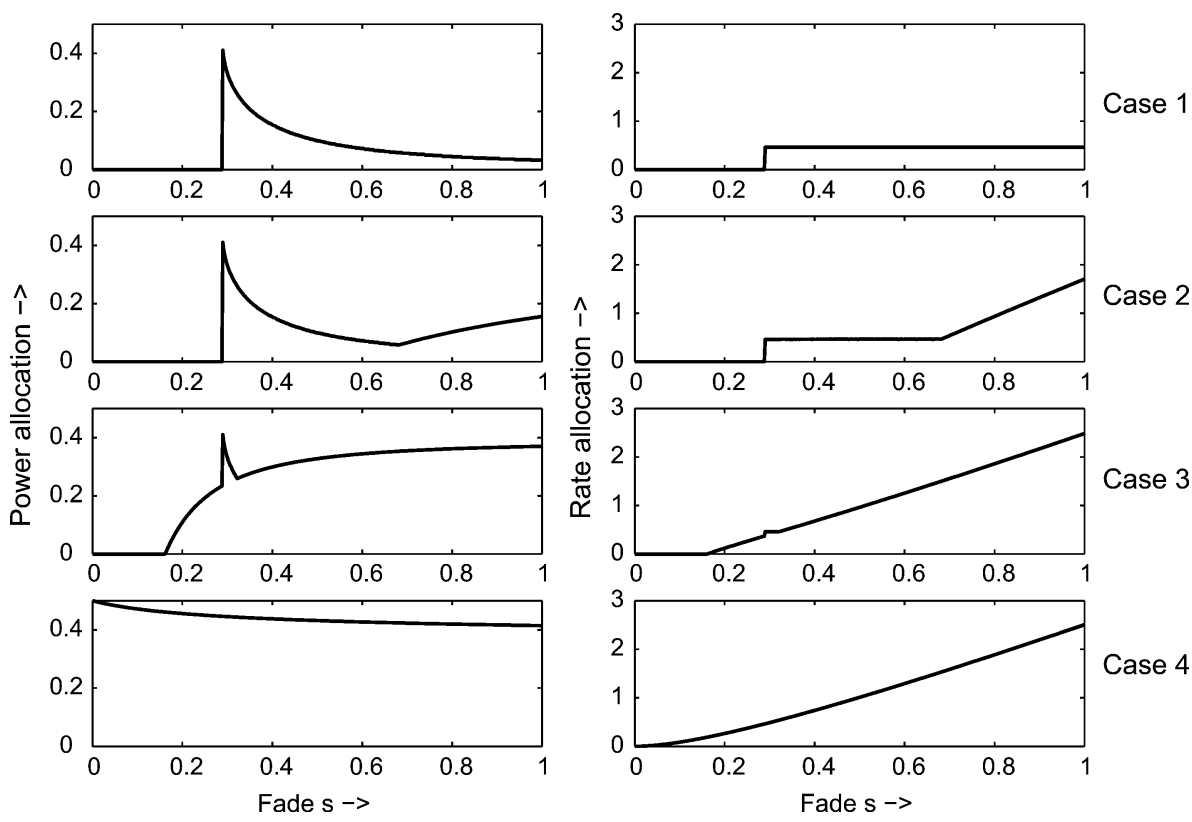

Fig. 6. Different types of optimal power and rate allocation schemes for the lognormal fading channel. The four cases correspond to different choices of $\sigma$. In Case 1 , we set $\sigma=\sigma_{\min }\left(r_{0}, \epsilon\right)=0.0585$. In Case 2, we choose $\sigma=0.1525$. In Case 3, we choose $\sigma=0.344$. In Case 4 , we choose $\sigma \geq \sigma_{0}=0.42$.

meet the basic rate requirement. When the second layer is required, the proportion of time this supplemental power is allocated is determined by the constraints on average power [c.f., (10)] and outage probability [c.f., (11)].

ii) The supplemental cost function $\Theta_{\text {supp }}(\cdot)$ partitions the channel state space into three disjoint sets: a) a service set, which is characterized by the condition $\Theta_{\text {supp }}\left(s, \rho_{1}^{*}\right)<\rho_{2}^{*}$, when the basic rate requirement is always met; b) a boundary set, characterized by $\Theta_{\text {supp }}\left(s, \rho_{1}^{*}\right)=\rho_{2}^{*}$, when a randomization between the two layers of power allocation is necessary; and c) an outage set, characterized by $\Theta_{\text {supp }}\left(s, \rho_{1}^{*}\right)>\rho_{2}^{*}$, when the supported rates are always below the basic rate requirement, resulting in service outage. The service set can further be partitioned into a basic rate set, when the supported rate is exactly equal to $r_{0}$, and an enhanced rate set, when the supported rate strictly exceeds $r_{0}$.

\section{Properties of the Optimal Power ALLOCATION SCHEME}

In this section, we discuss several properties of the optimal rate and power allocation scheme. From Theorem 1, it follows that the optimal solution is a combination of the ergodic capacity-achieving power allocation and the supplemental (basic rate) power allocation in the service set, and is randomized between these two deterministic power allocations in the boundary set. The optimal solution can be classified into four cases, depending on the values of $\left(\sigma, r_{0}, \epsilon\right)$. Note that in all of these cases, the feasibility of Problem 1 is assumed, i.e., (38) and (39) are satisfied. In Fig. 6, the optimal power allocation schemes and corresponding rate allocations are shown for each of these four cases.

Case 1: When $\sigma=\sigma_{\min }\left(r_{0}, \epsilon\right)$, i.e., the basic rate power allocation is just enough to meet the outage requirement, the optimal solution is the same as the power allocation that achieves outage capacity. In this case, $\Gamma^{*}(s)=\gamma_{\text {out }}^{*}(s)$, where $\gamma_{\text {out }}^{*}$ is the outage capacity-achieving power allocation scheme described in (36) and (37).

Case 2: When $\sigma>\sigma_{\min }\left(r_{0}, \epsilon\right)$ and $\left[\gamma_{\rho_{1}^{*}}(s)\right]^{+}=0$ in the outage set, the outage set is the same as that for the outage capacity calculation. In this case, the optimal solution includes no transmission in the outage set, a probabilistic power allocation in the boundary, a basic rate allocation in the basic rate set, and ergodic capacity allocation with rates larger than $r_{0}$ in the enhanced rate set.

Case 3: When $\sigma_{\min }\left(r_{0}, \epsilon\right)<\sigma<\sigma_{0}$ but $\left[\gamma_{\rho_{1}^{*}}(s)\right]^{+}>0$ in the outage set, rates lower than basic rate can be supported in the outage set. This case includes ergodic capacity power allocation with rates smaller than $r_{0}$ in the outage set, basic rate allocation in the basic rate set, and ergodic capacity power allocation with rates larger than $r_{0}$ in the enhanced rate set. Randomization between the basic rate allocation and ergodic capacity power allocation is done only in the boundary points.

Case 4: When $\operatorname{Pr}\left(I\left(\gamma_{\text {erg }}^{*}(S), S\right)\right) \geq 1-\epsilon$, then the ergodic capacity power allocation satisfies the outage requirement. In this case, the optimal power allocation comprises only the ergodic capacity power allocation. A further special case is when $\sigma \geq \sigma_{0}$, i.e., the average power constraint is inactive. In this case, the ergodic capacity power allocation is optimal, and no randomization is necessary. The outage set in this case is the outage floor set.

As $\sigma$ increases from $\sigma_{\min }\left(r_{0}, \epsilon\right)$ to $\sigma_{0}$, the optimal power allocation gradually changes from Case 1 to Case 4 . The serviceoutage-based achievable rate gradually changes from $r_{0}(1-\epsilon)$ to the ergodic capacity. Case 1 solution is the outage capacity power allocation, when the available power $\sigma=\sigma_{\min }\left(r_{0}, \epsilon\right)$ is just enough to satisfy the service outage constraint. As more power is available beyond $\sigma_{\min }\left(r_{0}, \epsilon\right)$, this additional power can be allocated in a more efficient manner to achieve higher average rates, while simultaneously satisfying the service outage 


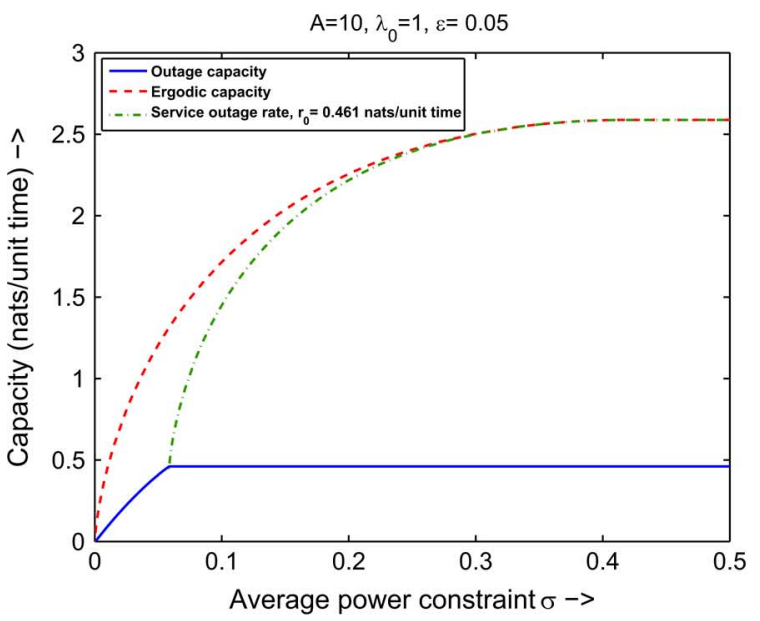

(a) Expected rates

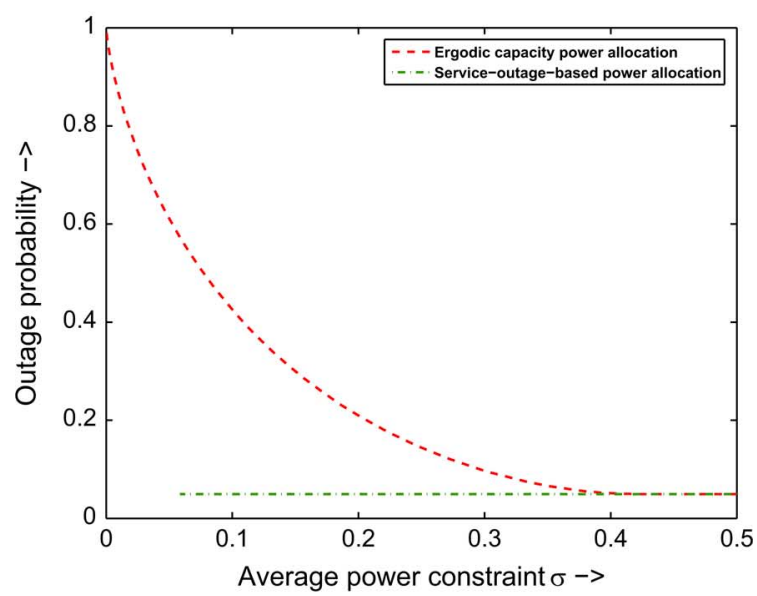

(b) Outage probability

Fig. 7. Comparison of (a) the service-outage-based achievable rate with ergodic and outage capacity; and (b) the outage probability for the service-outage-based power allocation with the ergodic capacity power allocation for a SISO Poisson fading channel with lognormal fading.

constraint. The additional power first allows an enhanced rate set (Case 2), and for higher values of $\sigma$, nonzero rates are supported in the outage set in addition to the enhanced rate set (Case 3). Finally, when $\sigma$ is large enough for the ergodic capacity power allocation to satisfy the service outage constraint, the ergodic allocation is optimal (Case 4). Thus, for fixed $\left(r_{0}, \epsilon\right)$, the optimal power allocation scheme gradually changes from the outage capacity power allocation to the ergodic capacity power allocation as $\sigma$ increases. For Cases $1-3$, the outage probability is exactly equal to $\epsilon$, while for Case 4 , the outage probability is less than $\epsilon$. The optimal solution is probabilistic at the boundary set only when $\rho_{2}^{*}>0$, i.e., for Cases $1-3$. The boundary set has a probability measure 0 for continuous fade distributions. In particular, the optimal power allocation is deterministic when the channel fade distribution is lognormal, as considered in the following example.

\section{NUMERICAL EXAMPLE}

Consider the SISO Poisson channel with lognormal fading from Example 2. Recall that $1 / 2 \log S \sim \mathcal{N}\left(\mu_{G}, \sigma_{G}^{2}\right)$, with $\sigma_{G}^{2}=-\mu_{G}=0.1$, and $A=10, \lambda_{0}=1$. The basic rate is fixed at $r_{0}=0.461$ nats/unit time, and the outage probability is fixed at $\epsilon=0.05$. We have seen in Example 5 that for this choice of $\left(r_{0}, \epsilon\right)$, Problem 1 has a solution provided that $\sigma \geq$ $\sigma_{\min }=0.0585$. In Fig. 6, we demonstrate the different cases for optimal power and rate allocation with the service-outage-based approach. The four cases correspond to different choices for $\sigma$. In Case 1, we set $\sigma=\sigma_{\min }\left(r_{0}, \epsilon\right)=0.0585$, whence the optimal power allocation is the same as outage optimal power allocation. In Case 2, we choose $\sigma=0.1525$. No rate is supported in the outage set (which is the same as in Case 1), but the additional power allows an enhanced rate set where rates higher than $r_{0}$ can be supported. In Case 3, we choose $\sigma=0.344$. The additional power not only allows an enhanced rate set as in Case 2 , but also allows rates lower than $r_{0}$ in the outage set with ergodic capacity power allocation. The basic rate power allocation is supported in an intermediate subset of channel states, sandwiched between the outage set and enhanced rate set. Finally, in Case 4 , we choose $\sigma \geq \sigma_{0}=0.42$. In this case, the ergodic capacity power allocation is optimal.

In Fig. 7(a), the service-outage-based achievable rates corresponding to a basic rate of $r_{0}=0.461$ nats/unit time are compared against ergodic and outage capacities for different values of $\sigma$. The service-outage-based power allocation scheme is feasible for $\sigma \geq \sigma_{\min }=0.0585$. Starting from $r_{0}(1-\epsilon)$, the service-outage-based rate allocation approaches the ergodic capacity as $\sigma$ increases. On the other hand, the outage capacity reaches the maximum $P_{0}^{-1}(\epsilon)=0.461$ nats/unit time for $\sigma=$ $\sigma_{L}(\epsilon)=0.0588$, which is significantly lower than the maximum ergodic capacity, $\mathbb{E}\left[I_{\max }(S)\right]=2.588$ nats/unit time. In Fig. 7(b), the outage probability corresponding to the ergodic capacity power allocation is compared with the serviceoutage-based power allocation scheme for different values of $\sigma$. There is a significant improvement in the outage performance for the service-outage-based power allocation scheme compared to the ergodic capacity. This example demonstrates that the service-outage-based approach has helped achieve higher expected rates while maintaining lower outage probabilities and thus effectively resolved these two conflicting objectives.

\section{CONCLUSION}

A service-outage-based probabilistic rate and power allocation problem for the SISO Poisson fading channel with perfect CSI at the transmitter and the receiver is considered. The objective of the allocation problem is to maximize the expected information transmission rate under an outage probability constraint in addition to the customary peak and average transmitter power constraints. Whenever the problem has a feasible solution, the optimal power allocation is shown to be a combination of the ergodic capacity power allocation and the basic rate power allocation with a randomization between the two deterministic allocations at the boundary points. The randomization is not necessary for the class of continuous fading distributions, e.g., lognormal fade. The service-outage-based approach judiciously resolves the conflicting objectives of high expected transmission rate and low outage probability. 
There are several interesting open problems, e.g., characterization of the service-outage-based rate and power allocation for 1) the MIMO Poisson fading channel; 2) the SISO Poisson fading channel with partial CSI at the transmitter; and 3) the Poisson fading channel with bandwidth constraints at the transmitter and the receiver. These topics are currently under investigation.

\section{APPENDIX I}

\section{PROOF OF PROPOSITION 2}

We closely follow the proof of [15, Lemma 1]. We demonstrate that for an arbitrary feasible probabilistic power allocation scheme $\Gamma(\cdot)$ with a conditional pdf $f_{\Gamma \mid S}$, it is possible to construct another feasible scheme $\Gamma^{\prime}(\cdot)$, which is randomized between two deterministic schemes $\gamma_{a}(\cdot)$ and $\gamma_{b}(\cdot)$ with $I\left(\gamma_{a}(s), s\right) \geq r_{0}$ and a timesharing factor $w(\cdot)$ satisfying $\mathbb{E}[w(S)] \geq 1-\epsilon$. Furthermore, the average rate achieved by $\Gamma^{\prime}(\cdot)$ is at least as much as the rate achieved by $\Gamma(\cdot)$, which implies that the optimal rate allocation is of the form (41).

Since $\Gamma(\cdot)$ is feasible, $\mathbb{E}[\Gamma(S)] \leq \sigma$ and $\operatorname{Pr}\{I(\Gamma(S), S)<$ $\left.r_{0}\right\} \leq \epsilon$ must hold. The deterministic power allocation schemes $\gamma_{a}(\cdot)$ and $\gamma_{b}(\cdot)$ and the timesharing factor $w(\cdot)$ are defined as follows:

$$
\begin{aligned}
w(s) & =\operatorname{Pr}\left\{I(\Gamma(S), S) \geq r_{0} \mid S=s\right\} \\
\gamma_{a}(s) & =\mathbb{E}\left[\Gamma(S) \mid I(\Gamma(S), S) \geq r_{0}, S=s\right] \\
\gamma_{b}(s) & =\mathbb{E}\left[\Gamma(S) \mid I(\Gamma(S), S)<r_{0}, S=s\right] .
\end{aligned}
$$

In other words, $\gamma_{a}(\cdot)$ is the conditional average of all the schemes in $\Gamma(\cdot)$ with rate at least $r_{0}, \gamma_{b}(\cdot)$ is the conditional average of all the schemes with rate less than $r_{0}$, and $w(\cdot)$ is the timesharing factor that specifies the proportion of time the rate is greater than or equal to the basic rate $r_{0}$ for a given fade. By definition [see (22)], for any $s \in \mathcal{S}_{0}\left(r_{0}\right)$, we must have $w(s)=0$. Furthermore, since $\Gamma(\cdot)$ meets the outage constraint (11), it follows that $\mathbb{E}[1-w(S)] \leq \epsilon$. Note that (38) is necessary to ensure that the outage constraint (11) is satisfied. Next, note that

$$
\begin{aligned}
I\left(\gamma_{a}(S), S\right) & =I\left(\mathbb{E}\left[\Gamma(S) \mid I(\Gamma(S), S) \geq r_{0}\right], S\right) \\
& \geq \mathbb{E}\left[I(\Gamma(S), S) \mid I(\Gamma(S), S) \geq r_{0}\right] \\
I\left(\gamma_{b}(S), S\right) & =I\left(\mathbb{E}\left[\Gamma(S) \mid I(\Gamma(S), S)<r_{0}\right], S\right) \\
& \geq \mathbb{E}\left[I(\Gamma(S), S) \mid I(\Gamma(S), S)<r_{0}\right]
\end{aligned}
$$

where (69) and (70) follow by the application of Jensen's inequality to the concave function $I(\cdot, s)$. From (69), we also get $I\left(\gamma_{a}(s), s\right) \geq r_{0}$ for any $s \in \mathcal{S}_{0}^{c}\left(r_{0}\right)$.

Consider now a probabilistic scheme $\Gamma^{\prime}(\cdot)$ such that

$$
\Gamma^{\prime}(s)= \begin{cases}\gamma_{a}(s), & \text { w. p. } w(s) \\ \gamma_{b}(s), & \text { w. p. } 1-w(s)\end{cases}
$$

The average power for $\Gamma^{\prime}(\cdot)$ is

$$
\begin{aligned}
\mathbb{E}\left[\Gamma^{\prime}(S)\right] & =\mathbb{E}\left[w(S) \gamma_{a}(S)+(1-w(S)) \gamma_{b}(S)\right] \\
& =\mathbb{E}[\Gamma(S)] \leq \sigma
\end{aligned}
$$

where (72) follows by (68). Since for any $s \in \mathcal{S}_{0}^{c}\left(r_{0}\right)$, $I\left(\gamma_{a}(s), s\right) \geq r_{0}$, and $\operatorname{Pr}\left\{\Gamma^{\prime}(s)=\gamma_{a}(s)\right\}=w(s)$, we have

$$
\operatorname{Pr}\left\{I\left(\Gamma^{\prime}(S), S\right) \geq r_{0} \mid S=s\right\} \geq w(s) .
$$

Thus, the outage probability of $\Gamma^{\prime}(\cdot)$ satisfies

$$
\begin{aligned}
\operatorname{Pr} & \left\{I\left(\Gamma^{\prime}(S), S\right)<r_{0}\right\} \\
& =1-\mathbb{E}\left[\operatorname{Pr}\left\{I\left(\Gamma^{\prime}(S), S\right) \geq r_{0} \mid S\right\}\right] \\
& \leq 1-\mathbb{E}[w(S)] \leq \epsilon .
\end{aligned}
$$

By (72) and (73), it follows that $\Gamma^{\prime}(\cdot)$ is also a feasible power allocation scheme for Problem 1. Furthermore, the average rate achieved by $\Gamma^{\prime}(\cdot)$ is

$$
\begin{aligned}
\mathbb{E} & {\left[I\left(\Gamma^{\prime}(S), S\right)\right] } \\
\quad & =\mathbb{E}\left[w(S) I\left(\gamma_{a}(S), S\right)+(1-w(S)) I\left(\gamma_{b}(S), S\right)\right] \\
& \geq \mathbb{E}[I(\Gamma(S), S)]
\end{aligned}
$$

where (74) follows by (69) and (70).

Thus, from any arbitrary feasible power allocation $\Gamma(\cdot)$, it is possible to construct another feasible power allocation scheme $\Gamma^{\prime}(\cdot)$, which is randomized between two deterministic power allocations, in the process achieving a higher average rate. Therefore, the optimal power allocation scheme must be of the form (41).

Finally, when $\sigma<\sigma_{0}$, at optimality $\mathbb{E}\left[\Gamma^{*}(S)\right]=\sigma$ must hold; otherwise, a higher average rate can be achieved by increasing the power.

\section{APPENDIX II}

\section{PROOF OF PROPOSITION 3}

We establish (57) first, followed by the proof of (56).

From (48) and (26), it follows that

$\frac{\partial L(\cdots)}{\partial \gamma_{b}^{*}(s)}=\left(1-w^{*}(s)\right)\left(s A \log \left(\frac{1+\xi\left(\frac{s A}{\lambda_{0}}\right) \frac{s A}{\lambda_{0}}}{1+\gamma_{b}^{*}(s) \frac{s A}{\lambda_{0}}}\right)-\rho_{1}^{*}\right)$

whence (50) implies that at optimality

$$
\begin{aligned}
\gamma_{b}^{*}(s) & =\frac{\lambda_{0}}{s A}\left[e^{-\left(1+\rho_{1}^{*} / s A\right)}\left(1+\frac{s A}{\lambda_{0}}\right)^{\left(1+\lambda_{0} / s A\right)}-1\right]^{+} \\
& =\left[\gamma_{\rho_{1}^{*}}(s)\right]^{+}
\end{aligned}
$$

by (17), which establishes (57).

From (48) and (26), we get

$\frac{\partial L(\cdots)}{\partial \gamma_{a}^{*}(s)}=\left(w^{*}(s)+u_{1}^{*}(s)\right) s A$

$$
\times \log \left(\frac{1+\xi\left(\frac{s A}{\lambda_{0}}\right) \frac{s A}{\lambda_{0}}}{1+\gamma_{a}^{*}(s) \frac{s A}{\lambda_{0}}}\right)-\rho_{1}^{*} w^{*}(s)
$$

whence by (49), for any $s \in \mathcal{S}_{0}^{c}\left(r_{0}\right)$, we get

$$
\begin{aligned}
\gamma_{a}^{*}(s)=\frac{\lambda_{0}}{s A}\left[e^{-\left(1+\rho_{1}^{*} w^{*}(s) / s A\left(w^{*}(s)+u_{1}^{*}(s)\right)\right)}\right. & \\
& \left.\times\left(1+\frac{s A}{\lambda_{0}}\right)^{\left(1+\lambda_{0} / s A\right)}-1\right]^{+} .
\end{aligned}
$$

When $u_{1}^{*}(s)=0$, then from (54), we have $I\left(\gamma_{a}^{*}(s), s\right) \geq$ $r_{0}$, and from (76), we have $\gamma_{a}^{*}(s)=\left[\gamma_{\rho_{1}^{*}}(s)\right]^{+}$. Therefore, in 
this case, we must have $I\left(\left[\gamma_{\rho_{1}^{*}}(s)\right]^{+}, s\right) \geq r_{0}$. On the other hand, when $u_{1}^{*}(s)>0$, from (54), we have $I\left(\gamma_{a}^{*}(s), s\right)=r_{0}$, i.e., $\gamma_{a}^{*}(s)=\gamma_{L}\left(s, r_{0}\right)$, while from (76), we have $\gamma_{a}^{*}(s) \leq$ $\left[\gamma_{\rho_{1}^{*}}(s)\right]^{+}$, which, by Proposition 1, implies that in this case $I\left(\left[\gamma_{\rho_{1}^{*}}(s)\right]^{+}, s\right) \leq r_{0}$. Summarizing these observations, we have for $s \in \mathcal{S}_{0}^{c}\left(r_{0}\right)$

$$
\gamma_{a}^{*}(s)= \begin{cases}{\left[\gamma_{\rho_{1}^{*}}(s)\right]^{+},} & \text {if } I\left(\left[\gamma_{\rho_{1}^{*}}(s)\right]^{+}, s\right) \geq r_{0} \\ \gamma_{L}\left(s, r_{0}\right), & \text { otherwise }\end{cases}
$$

which establishes (56).

\section{APPENDIX III \\ PROOF OF PROPOSITION 4}

First Part: The proof of the first part entails the establishment of the nonincreasing property of $\Theta_{\text {supp }}(\cdot, \rho)$. For any $s \in \mathcal{S}_{0}^{c}\left(r_{0}\right)$, when $\gamma_{\text {supp }}(s, \rho)=0$, we have $\gamma_{L}\left(s, r_{0}\right) \leq$ $\left[\gamma_{\rho}(s)\right]^{+}$, so that by Proposition $1, I\left(\left[\gamma_{\rho}(s)\right]^{+}, s\right) \geq r_{0}$, and hence, $I_{\text {supp }}(s, \rho)=0$. Therefore, $\Theta_{\text {supp }}(s, \rho)=0$ when $\gamma_{\text {supp }}(s, \rho)=0$.

On the other hand, for any $s \in \mathcal{S}_{0}^{c}\left(r_{0}\right)$, when $\gamma_{\text {supp }}(s, \rho)>0$, we have

$$
\begin{aligned}
& \gamma_{\text {supp }}(s, \rho)=\gamma_{L}\left(s, r_{0}\right)-\left[\gamma_{\rho}(s)\right]^{+} \\
& I_{\text {supp }}(s, \rho)=r_{0}-I\left(\left[\gamma_{\rho}(s)\right]^{+}, s\right)
\end{aligned}
$$

so that

$$
\Theta_{\text {supp }}(s, \rho)=\rho \gamma_{L}\left(s, r_{0}\right)-r_{0}-q(s, \rho)
$$

with

$$
q(s, \rho) \triangleq \rho\left[\gamma_{\rho}(s)\right]^{+}-I\left(\left[\gamma_{\rho}(s)\right]^{+}, s\right) .
$$

To establish the nonincreasing property of $\Theta_{\text {supp }}(\cdot, \rho)$, by (78), we need to show that

$$
\frac{\partial \Theta_{\text {supp }}}{\partial s}=\rho \frac{\partial \gamma_{L}}{\partial s}-\frac{\partial q}{\partial s} \leq 0
$$

when $\left[\gamma_{\rho}(s)\right]^{+}<\gamma_{L}\left(s, r_{0}\right)$.

From (17), it can be verified by simple algebra that

$$
\zeta\left(s A, \lambda_{0}\right)-s A\left(1+\log \left(\gamma_{\rho}(s) s A+\lambda_{0}\right)\right)=\rho
$$

whence it follows that

$$
\frac{\partial \gamma_{\rho}(s)}{\partial s}=\frac{-\gamma_{\rho}(s)}{s}+\frac{\gamma_{\rho}(s) s A+\lambda_{0}}{s^{2} A} \log \left(\frac{1+\frac{s A}{\lambda_{0}}}{1+\gamma_{\rho}(s) \frac{s A}{\lambda_{0}}}\right) \text {. }
$$

From (3) and (81), it follows that

$$
\frac{d I\left(\gamma_{\rho}(s), s\right)}{d s}=\gamma_{\rho}(s) A \log \left(\frac{1+\frac{s A}{\lambda_{0}}}{1+\gamma_{\rho}(s) \frac{s A}{\lambda_{0}}}\right)+\rho \frac{\partial \gamma_{\rho}(s)}{\partial s}
$$

whence by (79) and (83), it follows that

$$
\frac{\partial q}{\partial s}= \begin{cases}-\gamma_{\rho}(s) A \log \left(\frac{1+\frac{s A}{\lambda_{0}}}{1+\gamma_{\rho}(s) \frac{s A}{\lambda_{0}}}\right), & \gamma_{\rho}(s)>0 \\ 0, & \gamma_{\rho}(s) \leq 0 .\end{cases}
$$

Since $\gamma_{\rho}(s) \leq 1$, it follows from (84) that $\partial q / \partial s \leq 0$. Differentiating both sides of the equation $I\left(\gamma_{L}, s\right)=r_{0}$ with respect to $s$, from (3) and (14), we get after some rearrangement

$$
\frac{\partial \gamma_{L}}{\partial s}=-\frac{\gamma_{L}\left(s, r_{0}\right)}{s} \frac{\log \left(\frac{1+\frac{s A}{\lambda_{0}}}{1+\gamma_{L}\left(s, r_{0}\right) \frac{s A}{\lambda_{0}}}\right)}{\log \left(\frac{1+\xi\left(\frac{s A}{\lambda_{0}}\right) \frac{s A}{\lambda_{0}}}{1+\gamma_{L}\left(s, r_{0}\right) \frac{s A}{\lambda_{0}}}\right)}
$$

which is nonpositive since $0 \leq \gamma_{L}\left(s, r_{0}\right) \leq \xi\left(s A / \lambda_{0}\right) \leq 1 / 2$ for any $s \in \mathcal{S}_{0}^{c}\left(r_{0}\right)$. From (84) and (85), it follows that when $\gamma_{\rho}(s) \leq 0, \partial \Theta_{\text {supp }} / \partial s \leq 0$, and in this case, $\Theta_{\text {supp }}(\cdot, \rho)$ is nonincreasing. When $\gamma_{\rho}(s)>0$, we have by (84) and (85)

$$
\begin{array}{r}
\rho \frac{\partial \gamma_{L}}{\partial s}-\frac{\partial q}{\partial s}=\gamma_{\rho}(s) A \log \left(\frac{1+\frac{s A}{\lambda_{0}}}{1+\gamma_{\rho}(s) \frac{s A}{\lambda_{0}}}\right) \\
-\rho \frac{\gamma_{L}\left(s, r_{0}\right)}{s} \frac{\log \left(\frac{1+\frac{s A}{\lambda_{0}}}{1+\gamma_{L}\left(s, r_{0}\right) \frac{s A}{\lambda_{0}}}\right)}{\log \left(\frac{1+\xi\left(\frac{s A}{\lambda_{0}}\right) \frac{s A}{\lambda_{0}}}{1+\gamma_{L}\left(s, r_{0}\right) \frac{s A}{\lambda_{0}}}\right)}
\end{array}
$$

with both the terms on the right-hand side above being nonnegative. When $\gamma_{L}\left(s, r_{0}\right)=\xi\left(s A / \lambda_{0}\right)=\gamma_{0}(s)$, i.e., when $s=s_{0}\left(r_{0}\right)=I_{\max }^{-1}\left(r_{0}\right)$, the second term on the right-hand side above is unbounded, while the first term is bounded, so that $\partial \Theta_{\text {supp }} / \partial s=-\infty$. Finally, using the identity [which follows from (81)]

$$
s A \log \left(\frac{1+\xi\left(\frac{s A}{\lambda_{0}}\right) \frac{s A}{\lambda_{0}}}{1+\gamma_{\rho}(s) \frac{s A}{\lambda_{0}}}\right)=\rho
$$

we can simplify the term

$\gamma_{\rho}(s) s A \log \left(\frac{1+\frac{s A}{\lambda_{0}}}{1+\gamma_{\rho}(s) \frac{s A}{\lambda_{0}}}\right) \log \left(\frac{1+\xi\left(\frac{s A}{\lambda_{0}}\right) \frac{s A}{\lambda_{0}}}{1+\gamma_{L}\left(s, r_{0}\right) \frac{s A}{\lambda_{0}}}\right)$ 


$$
\begin{aligned}
- & \rho \gamma_{L}\left(s, r_{0}\right) \log \left(\frac{1+\frac{s A}{\lambda_{0}}}{1+\gamma_{L}\left(s, r_{0}\right) \frac{s A}{\lambda_{0}}}\right) \\
= & \gamma_{\rho}(s)\left\{\rho+s A \log \left(\frac{1+\gamma_{\rho}(s) \frac{s A}{\lambda_{0}}}{1+\gamma_{L}\left(s, r_{0}\right) \frac{s A}{\lambda_{0}}}\right)\right\} \\
& \times\left\{\log \left(\frac{1+\frac{s A}{\lambda_{0}}}{1+\gamma_{L}\left(s, r_{0}\right) \frac{s A}{\lambda_{0}}}\right) \log \left(\frac{1+\gamma_{\rho}(s) \frac{s A}{\lambda_{0}}}{1+\gamma_{L}\left(s, r_{0}\right) \frac{s A}{\lambda_{0}}}\right)\right\} \\
& -\rho \gamma_{L}\left(s, r_{0}\right) \log \left(\frac{s A}{1+\gamma_{L}\left(s, r_{0}\right) \frac{s A}{\lambda_{0}}}\right) \\
= & -\rho\left(\gamma_{L}(s, \rho)-\gamma_{\rho}(s)\right) \log \left(\frac{s A}{1+\gamma_{L}\left(s, r_{0}\right) \frac{s A}{\lambda_{0}}}\right) \\
& \times \log \left(\frac{1+\frac{s A}{\lambda_{0}}}{1+\xi\left(\frac{s A}{\lambda_{0}}\right) \frac{s A}{\lambda_{0}}}\right) \\
& \left(\frac{1+\gamma_{L}\left(s, r_{0}\right) \frac{s A}{\lambda_{0}}}{1+\gamma_{\rho}(s) \frac{s A}{\lambda_{0}}}\right) \\
& (8 \log )
\end{aligned}
$$

whence by (80), (86), and (88), we get

$$
\begin{aligned}
\frac{\partial \Theta_{\text {supp }}}{\partial s}= & \rho \frac{\partial \gamma_{L}}{\partial s}-\frac{\partial q}{\partial s} \\
= & -\rho\left(\gamma_{L}(s, \rho)-\gamma_{\rho}(s)\right) \frac{\log \left(\frac{1+\frac{s A}{\lambda_{0}}}{1+\gamma_{L}\left(s, r_{0}\right) \frac{s A}{\lambda_{0}}}\right)}{\log \left(\frac{1+\xi\left(\frac{s A}{\lambda_{0}}\right) \frac{s A}{\lambda_{0}}}{1+\gamma_{L}\left(s, r_{0}\right) \frac{s A}{\lambda_{0}}}\right)} \\
& -\gamma_{\rho}(s) s A \frac{\log \left(\frac{1+\gamma_{L}\left(s, r_{0}\right) \frac{s A}{\lambda_{0}}}{1+\gamma_{\rho}(s) \frac{s A}{\lambda_{0}}}\right)}{\log \left(\frac{1+\xi\left(\frac{s A}{\lambda_{0}}\right) \frac{s A}{\lambda_{0}}}{1+\gamma_{L}\left(s, r_{0}\right) \frac{s A}{\lambda_{0}}}\right)} \\
& \times \log \left(\frac{\left.1+\frac{s A}{\lambda_{0}}\right)}{\left.1+\xi\left(\frac{s A}{\lambda_{0}}\right) \frac{s A}{\lambda_{0}}\right)}\right.
\end{aligned}
$$

and observe that the right-hand side of (89) is negative when $\gamma_{\rho}(s)<\gamma_{L}\left(s, r_{0}\right)<\xi\left(s A / \lambda_{0}\right) \leq \frac{1}{2}$. This completes the proof of the first part of Proposition 4.
Second Part: We have already established that $\Theta_{\text {supp }}(s, \rho)=0$ when $\gamma_{\text {supp }}(s, \rho)=0$. It remains to show that $\Theta_{\text {supp }}(s, \rho)>0$ when $\gamma_{\text {supp }}(s, \rho)>0$, i.e., when $\gamma_{L}\left(s, r_{0}\right)>\left[\gamma_{\rho}(s)\right]^{+}$. In this case, noting that $r_{0}=I\left(\gamma_{L}\left(s, r_{0}\right), s\right)>I\left(\left[\gamma_{\rho}(s)\right]^{+}, s\right)$, from (63), we get

$$
\begin{aligned}
& \Theta_{\text {supp }}(s, \rho) \\
& =\rho\left(\gamma_{L}\left(s, r_{0}\right)-\left[\gamma_{\rho}(s)\right]^{+}\right)-I\left(\gamma_{L}\left(s, r_{0}\right), s\right)+I\left(\left[\gamma_{\rho}(s)\right]^{+}, s\right) \\
& =\rho\left(\gamma_{L}\left(s, r_{0}\right)-\left[\gamma_{\rho}(s)\right]^{+}\right)-\gamma_{L}\left(s, r_{0}\right) s A \\
& \times \log \left(\frac{1+\xi\left(\frac{s A}{\lambda_{0}}\right) \frac{s A}{\lambda_{0}}}{1+\xi\left(\frac{\gamma_{L}\left(s, r_{0}\right) s A}{\lambda_{0}}\right) \frac{\gamma_{L}\left(s, r_{0}\right) s A}{\lambda_{0}}}\right)+\left[\gamma_{\rho}(s)\right]^{+} s A \\
& \times \log \left(\frac{1+\xi\left(\frac{s A}{\lambda_{0}}\right) \frac{s A}{\lambda_{0}}}{1+\xi\left(\frac{\left[\gamma_{\rho}(s)\right]^{+} s A}{\lambda_{0}}\right) \frac{\left[\gamma_{\rho}(s)\right]^{+} s A}{\lambda_{0}}}\right) \\
& =-\left(\gamma_{L}\left(s, r_{0}\right)-\left[\gamma_{\rho}(s)\right]^{+}\right) s A \log \left(1+\gamma_{\rho}(s) \frac{s A}{\lambda_{0}}\right)+\gamma_{L}\left(s, r_{0}\right) s A \\
& \times \log \left(1+\xi\left(\frac{\gamma_{L}\left(s, r_{0}\right) s A}{\lambda_{0}}\right) \frac{\gamma_{L}\left(s, r_{0}\right) s A}{\lambda_{0}}\right)-\left[\gamma_{\rho}(s)\right]^{+} s A \\
& \times \log \left(1+\xi\left(\frac{\left[\gamma_{\rho}(s)\right]^{+} s A}{\lambda_{0}}\right) \frac{\left[\gamma_{\rho}(s)\right]^{+} s A}{\lambda_{0}}\right)
\end{aligned}
$$

where (90) follows by (15) and (91) follows by (87).

We denote the right-hand side of (91) as $H\left(\gamma_{L}\left(s, r_{0}\right), \quad\left[\gamma_{\rho}(s)\right]^{+}\right)$, which is equal to zero when $\gamma_{L}\left(s, r_{0}\right)=\left[\gamma_{\rho}(s)\right]^{+}$. It remains to show that $H\left(\gamma_{L}\left(s, r_{0}\right),\left[\gamma_{\rho}(s)\right]^{+}\right)>0$ when $\gamma_{L}\left(s, r_{0}\right)>\left[\gamma_{\rho}(s)\right]^{+}$. This can be easily accomplished by showing that

$$
\frac{\partial H}{\partial \gamma_{L}}=s A \log \left(\frac{1+\gamma_{L}\left(s, r_{0}\right) \frac{s A}{\lambda_{0}}}{1+\gamma_{\rho}(s) \frac{s A}{\lambda_{0}}}\right)
$$

which is clearly positive when $\gamma_{L}\left(s, r_{0}\right)>\left[\gamma_{\rho(s)}\right]^{+}$. Hence, $H$ is an increasing function for $\gamma_{L}\left(s, r_{0}\right)>\left[\gamma_{\rho}(s)\right]^{+}$implying $H\left(\gamma_{L}\left(s, r_{0}\right),\left[\gamma_{\rho}(s)\right]^{+}\right)>0$ when $\gamma_{L}\left(s, r_{0}\right)>\left[\gamma_{\rho}(s)\right]^{+}$. This concludes the proof.

\section{ACKNOWLEDGMENT}

The authors would like to thank the anonymous reviewers and the associate editor for their thorough reviews and constructive comments.

\section{REFERENCES}

[1] G. Caire, G. Taricco, and E. Biglieri, "Optimum power control over fading channels," IEEE Trans. Inf. Theory, vol. 45, no. 5, pp. 1468-1489, Jul. 1999.

[2] K. Chakraborty and P. Narayan, "The Poisson fading channel," IEEE Trans. Inform. Theory, vol. 53, no. 7, pp. 2349-2364, Jul. 2007.

[3] K. Chakraborty, "Capacity of the MIMO optical fading channel," in Proc. Int. Symp. Inf. Theory, Adelaide, Australia, Sep. 2005, pp. 530-534.

[4] K. Chakraborty, S. Dey, and M. Franceschetti, "Outage capacity of MIMO Poisson fading channels," IEEE Trans. Inf. Theory, vol. 54, no. 11, pp. 4887-4907, Nov. 2008.

[5] M. H. A. Davis, "Capacity and cutoff rate for Poisson-type channels," IEEE Trans. Inf. Theory, vol. IT-26, no. 6, pp. 710-715, Nov. 1980. 
[6] A. J. Goldsmith and P. Varaiya, "Capacity of fading channels with channel side information," IEEE Trans. Inf. Theory, vol. 43, no. 6, pp. 1986-1992, Nov. 1997.

[7] S. M. Haas and J. H. Shapiro, "Capacity of wireless optical communications," IEEE J. Sel. Areas Commun., vol. 21, no. 8, pp. 1346-1357, Oct. 2003.

[8] S. Hranilovic and F. R. Kschischang, "Capacity bounds for power- and band-limited optical intensity channels corrupted by Gaussian noise," IEEE Trans. Inf. Theory, vol. 50, no. 5, pp. 784-795, May 2004.

[9] A. Ishimaru, Wave Propagation and Scattering in Random Media. New York: IEEE Press/Oxford Univ. Press, 1997.

[10] R. Knopp and P. A. Humblet, "On coding for block fading channels," IEEE Trans. Inf. Theory, vol. 46, no. 1, pp. 189-205, Jan. 2000.

[11] E. J. Lee and V. W. S. Chan, "Part 1: optical communication over the clear turbulent atmospheric channel using diversity," IEEE J. Sel. Areas Commun., vol. 22, no. 9, pp. 1896-1906, Nov. 2004.

[12] J. Li and M. Uysal, "Achievable information rate for outdoor free space optical communication with intensity modulation and direct detection," in Proc. IEEE Global Commun. Conf., San Francisco, CA, Nov. 2003, pp. 2654-2658.

[13] D. G. Luenberger, Optimization by Vector Space Methods. New York: Wiley, 1968.

[14] J. Luo, L. Lin, R. Yates, and P. Spasojevic, "Service outage based power and rate allocation," IEEE Trans. Inf. Theory, vol. 49, no. 1, pp. 323-330, Jan. 2003.

[15] J. Luo, R. Yates, and P. Spasojevic, "Service outage based power and rate allocation for parallel fading channels," IEEE Trans. Inf. Theory, vol. 51, no. 7, pp. 2594-2611, Jul. 2005.

[16] J. Proakis, E. Biglieri, and S. Shamai, "Fading channels: Informationtheoretic and communications aspects," IEEE Trans. Inf. Theory, vol. 44, no. 6, pp. 2619-2692, Oct. 1998.

[17] S. Shamai, "On the capacity of a direct-detection photon channel with intertransition-constrained binary input," IEEE Trans. Inf. Theory, vol. 37, no. 6, pp. 1540-1550, Nov. 1991.

[18] S. Shamai and A. Lapidoth, "Bounds on the capacity of a spectrally constrained Poisson channel," IEEE Trans. Inf. Theory, vol. 39, no. 1 , pp. 19-29, Jan. 1993.

[19] H. A. Willebrand and B. S. Ghuman, "Fiber optics without fiber," IEEE Spectrum, vol. 38, no. 8, pp. 41-45, Aug. 2001.

[20] S. G. Wilson, M. Brandt-Pierce, Q. Cao, and J. Levesque, "Free-space optical MIMO communication with $Q$-ary PPM," IEEE Trans. Commun., vol. 53, no. 8, pp. 1402-1412, Aug. 2005.

[21] X. Zhu and J. M. Kahn, "Free-space optical communication through atmospheric turbulent channels," IEEE Trans. Commun., vol. 50, no. 8, pp. 1293-1300, Aug. 2002.

Kaushik Chakraborty (SM'99-M'05) received the B.Tech. degree in electronics and electrical communication engineering from Indian Institute of Technology, Kharagpur, India, in 1998 and the M.S. and Ph.D. degrees in electrical and computer engineering from the University of Maryland, College Park, in 2000 and 2005, respectively.

From 2005 to 2008, he was a Postdoctoral Scholar at the California Institute of Telecommunications and Information Technology (CalIT2), University of Cali- fornia, San Diego. He is currently a Senior Systems Engineer at Qualcomm Inc., San Diego, CA. His research interests are in the areas of communication, signal processing, and information theory, with applications to broadband wireless and mobile communication networks.

Subhrakanti Dey (S'94-M'96-SM'06) was born in Calcutta, India, in 1968. He received the B.Tech. and M.Tech. degrees from the Department of Electronics and Electrical Communication Engineering, Indian Institute of Technology, Kharagpur, India, in 1991 and 1993, respectively, and the Ph.D. degree from the Department of Systems Engineering, Research School of Information Sciences and Engineering, Australian National University, Canberra, Australia, in 1996.

He has been with the Department of Electrical and Electronic Engineering, University of Melbourne, Parkville, Vic., Australia, since February 2000, where he is currently a full Professor. From September 1995 to September 1997 and September 1998 to February 2000, he was a postdoctoral Research Fellow with the Department of Systems Engineering, Australian National University. From September 1997 to September 1998, he was a Postdoctoral Research Associate with the Institute for Systems Research, University of Maryland, College Park. His current research interests include signal processing for telecommunications, wireless communications and networks, performance analysis of communication networks, stochastic and adaptive estimation and control, and statistical and adaptive signal processing.

Dr. Dey currently serves on the Editorial Board of the IEEE TRANSACTIONS on Automatic Control, IEEE TRANSACtions on Signal Processing, and Elsevier Systems and Control Letters.

Massimo Franceschetti (M'98) received the Laurea degree (magna cum laude) in computer engineering from the University of Naples in 1997, and the M.S. and $\mathrm{Ph}$.D. degrees in electrical engineering from the California Institute of Technology (Caltech), Pasadena, CA, in 1999 and 2003, respectively.

Currently, he is an Associate Professor of Electrical and Computer Engineering at the University of California at San Diego (UCSD). Before joining UCSD, he was a Postdoctoral Scholar at University of California at Berkeley for two years. He has held visiting positions at the Vrije Universiteit, Amsterdam, The Netherlands, the Ecole Polytechnique Federale de Lausanne, Switzerland, and the University of Trento, Italy. His research interests are in communication systems theory and include random networks, wave propagation in random media, wireless communication, and control over networks.

Prof. Franceschetti was awarded the C. H. Wilts Prize in 2003 for best doctoral thesis in electrical engineering at Caltech; the S. A. Schelkunoff award in 2005 (jointly with Profs. J. Bruck and L. J. Shulman) for best paper in the IEEE TRANSACTIONS ON ANTENNAS AND PROPAGation; a National Science Foundation (NSF) CAREER award in 2006, and an Office of Naval Research (ONR) Young Investigator award in 2007. He was on the guest editorial board of the IEEE TRANSACTIONS ON INFORMATION THEORY, Special Issue on Models, Theory, and Codes, for Relaying and Cooperation in Communication Networks; and of the IEEE Journal ON SELECTED AREAS IN COMmunications, Special Issue on Control and Communications. 